\section{Adaptive Joint Source-Channel Rate Allocation Policies for Delay Sensitive Applications Over Fading Channels}

\author{
Chandrashekhar Thejaswi PS and \\ Vinod Sharma, Senior Member, IEEE
}

\begin{abstract}
In this correspondence, we consider a problem of optimal source, channel coding rate pair allocation over a wireless link based on the channel fading state and the queue length of the transmitter. The source is delay sensitive. Therefore, our objective is to minimize the mean delay under given mean power and distortion constraints. We show that by jointly optimizing the source and channel rates, one can gain substantially in performance as compared to only source or channel rate optimization, as is usually considered in the literature. This is shown for single user, single-input-single-output (SISO), multiple-input-multiple-output (MIMO), and multiuser systems. The methods used are from Markov decision theory.
\end{abstract}

Index Terms-Joint source-channel coding, Markov decision theory, mean distortion constraint, multiple-input-multiple-output (MIMO) systems, multiple-access channel, wireless link.

\section{INTRODUCTION}

In this correspondence, we consider the problem of transmitting an analog/discrete source over a noisy fading channel. By Shannon's theorem [14], if the source and the channel are information stable, one can independently design a source encoder and a channel encoder without any loss of performance in distortion. However, in general it requires arbitrarily long source and channel codes. For delay sensitive applications, the resulting delay can be intolerable. Thus, for acceptable delay and mean distortion, it may be advantageous to use a joint source-channel encoder and decoder. But an optimal joint source-channel encoder depends upon the specific source and channel characteristics. However, in practical systems, one may want to use common channel encoders for different sources and applications. Therefore, as a compromise, one also considers systems where different sources have source encoders while a set of common channel encoders are used. Out of various combinations of source and channel encoders, one may pick one that is good for a specific source-channel pair [7], [8]. It is shown in [8] that such a system can provide significant reduction in mean distortion over fading channels. In this correspondence, we consider this problem in more detail.

We consider the transmission over a slowly fading channel. The system is slotted. The fading process is constant over a slot. The fading varies from slot to slot as a discrete time Markov chain with continuous state space. The channel state is available at the transmitter and the receiver. The source output generated in a slot is quantized, coded and stored in a buffer. The channel encoder picks a number of bits from the buffer, encodes them and transmits on the channel. Depending upon the channel state and the buffer contents appropriate rates for the source encoder and the channel encoder are chosen. We obtain rate policies

Manuscript received December 18, 2004; revised April 19, 2006. This work was supported in part by the DRDO-IISc Program on Advanced Research in Mathematical Engineering. The material in this correspondence was presented in part at the IEEE International Symposium on Information Theory Chicago, IL, June/July 2004.

C. Thejaswi PS is with the Research and Technology Division of Honeywell Technology Solutions Lab, Bangalore, India (e-mail: c_thejaswi@yahoo.com).

V. Sharma is with the Department of Electrical Communication Engineering, Indian Institute of Science, Bangalore, India (e-mail: vinod@ece.iisc.ernet.in).

Communicated by V. A. Vaishampayan, Associate Editor At Large.

Digital Object Identifier 10.1109/TIT.2006.883635 which minimize the mean delay in the buffer while keeping the mean distortion and the average transmit power within certain bounds.

In the following, we survey the related work. The study [8] mentioned above is closest to our approach. However, they study a discrete channel (with no average power constraint) and the queuing delays are not considered. Furthermore, they obtain optimal source and channel rates for each channel state by exhaustive search over a finite number of possibilities. In our setup, because of buffering, Markov modeled channel fading process and long term average delay, distortion and power constraints there is dependence in the source-channel rates picked in different slots. Thus, we address the problem via Markov decision theory.

The set up considered in [1], [4] is similar to ours. But there is no adaptive source coding. In [2], sequential quantization of a Markov source is considered. They also work in Markov decision theory framework and minimize the entropy of the quantized source with an upper bound on the mean distortion. However, there is no channel involved and no queuing delays are considered. Kashyap et al. [9] considers linear coding and decoding techniques to minimize mean distortion of transmitting a Gaussian source over a multi-antenna Rayleigh/Rician-fading channel. There is average power constraint but queuing delays are not considered. The work in [3] minimizes the average transmit power while maintaining an upper bound on the average distortion. The channel coding and modulation are fixed and there is no buffering.

The correspondence is organized as follows. In Section II-A, we propose the model for our system and state the assumptions. We formulate the problem as a constrained optimization problem. This is converted into a family of unconstrained problems via the Lagrangian approach. In Section II-B, we formulate this unconstrained problem as an average cost Markov Decision Process (MDP). In Section III, we show the existence of stationary average cost optimal policies which can be obtained as a limit of discounted cost optimal policies. We also show that these optimal policies are optimal for the original unconstrained problem. In Section III-B, we obtain structural results for the discounted optimal cost and policies some of which are needed in Section III-A. In Section III-C, we illustrate our optimal policy on an example and also compare its cost with the policies available in literature. We find that the optimal policy obtained here can provide significant performance improvement. In Sections IV and V, we extend our work to multiple-input-multiple-output (MIMO) and multiuser cases, respectively.

\section{SINGLE USER, SISO SYSTEMS}

In this section, we consider one source. The transmitter and the receiver have one antenna each. Generalizations to multiple antennas and multiuser systems will be considered later. In Section II-A, we explain the model and formulate the problem. In Section II-B, we show that this problem can be posed in the standard Markov Decision Theory setup. In Section III, we will study this problem in detail.

\section{A. System Model}

We consider a slotted system with a single user communicating over a frequency nonselective fading wireless channel. The fading stays constant during a slot. The data to be transmitted, arrives from a higher layer. This data is subjected to a variable rate compression depending on delay, power and distortion requirements. This compressed data is placed in a buffer of infinite capacity for transmission over the fading channel.

The transmitter acts as a controller which, with the knowledge of the buffer and the channel states, obtains optimum source and channel coding rates and transmission power. The objective is to minimize the 
mean delay subject to long run average transmit power and distortion constraints. Based on these decisions, the transmitter compresses the data, performs channel coding, and transmits over the channel.

In a practical system, e.g., for transmission of voice/video, the overall goal is to be able to transmit with an upper bound on the end to end distortion (mean square error) for an acceptable performance. To reduce the complexity of the problem, this upper bound can be split into two components: an upper bound on the mean square distortion for source coding and an upper bound on the bit error rate (BER) at the receiver channel decoder. A judicious choice of this split can provide a reasonable solution for the overall system design. In our setup, we assume that this has been done.

We consider the problem under the following set of assumptions.

- The data transmitted on the channel experiences fading and is also corrupted by additive noise. The fading gain is $G_{n}$ in slot $n$. The fading process $\left\{G_{n}\right\}$ is assumed to be a homogeneous Markov process with transition function $P_{G}$ with a compact state space $\mathcal{G}:=[\bar{g}, \bar{G}]$. We assume that it is Harris recurrent (with respect to a finite measure on its state space). The mean time $E_{g}\left[\tau_{\bar{G}}\right]$ to reach the state $\bar{G}$ from any state $g$, is finite. The fading process is also stochastically nondecreasing, i.e., $E\left[f\left(G_{n+1}\right) \mid G_{n}=g\right]$ is nondecreasing in $g$ if $f$ is a nondecreasing function. This indicates positive correlation in the process and hence should be generally satisfied. We also assume that $P_{G}\left(G_{n+1} \in B \mid G_{n}=g\right)$ is continuous in $g$ for every measurable $B$.

- The data input $B_{n}$ in slot $n$ to the source encoder is iid belonging to an analog/discrete alphabet $\zeta$ with distribution $P_{\zeta}$ and variance $\sigma_{\zeta}^{2}$

- Source outputs $J$ symbols per slot and there are $N$ channel uses in one slot.

- The number of bits arriving into the buffer in each slot is upper bounded by a positive number $\bar{A}, \bar{A}<\infty$.

- Channel and buffer state information are available at the transmitter and the receiver. The channel gain remains constant during a slot.

- $P(g, u)$, the power needed to transmit $u$ bits in a slot when the channel gain $g$ is nondecreasing in $u$ and nonincreasing and lower semicontinuous in $g$. Also, $P(g, u)$ is convex in $u$ (needed only for the Propositions 3 and 4).

The following are common examples that satisfy our assumptions. - Consider a Gaussian channel with fading. Then, the power function is

$$
P(g, u)=\frac{\sigma_{w}^{2}}{|g|^{2}}\left(e^{k u}-1\right)
$$

where $k=\frac{2 \ln (2)}{N}$ and $\sigma_{w}^{2}$ is the noise variance.

- Consider the above channel with uncoded BPSK modulation. Then, the BER (bit error rate) is given by

$$
P_{e}=Q\left(\sqrt{2|g|^{2} \frac{P(g, u)}{\sigma_{w}^{2} u}}\right) .
$$

Thus, the power expended for a target BER of $P_{e}$ is

$$
P(g, u)=\frac{u}{2 \cdot|g|^{2}} \sigma_{w}^{2}\left(Q^{-1}\left(P_{e}\right)\right)^{2} .
$$

With appropriate sampling, one can represent the channel output at time $n$ as

$$
Y_{n}=G_{n} Z_{n}+W_{n}, \quad n=0,1,2, \ldots
$$

where $Z_{n}$ is the transmitted symbol and $W_{n}$ is the additive noise process at time $n$.
Let $S_{n}$ be the amount of data in the buffer at the beginning of the $n^{\text {th }}$ slot and $S_{n} \in \mathcal{S}$ where $\mathcal{S}=\{0,1,2, \ldots\}$. We define the state of the system in the $n^{\text {th }}$ slot as $X_{n}:=\left(S_{n}, G_{n}\right)$. At the $n^{\text {th }}$ decision epoch, the transmitter decides upon the amount of data bits $A_{n}$ to be placed in the buffer and the amount of data bits $U_{n}$ to be removed from the buffer for transmission. The evolution of the buffer state process is given by

$$
S_{n+1}=S_{n}+A_{n}-U_{n}
$$

where

$$
U_{n} \in\left\{0,1, \ldots, S_{n}\right\} \text { and } A_{n} \in \mathcal{A}:=\{0,1, \ldots, \bar{A}\}, \bar{A}<\infty .
$$

For the $n$th slot the transmitter incurs a cost of

- distortion $D\left(\frac{A_{n}}{J}\right)$ to encode $J$ source symbols into $A_{n}$ bits where $D($.$) is the distortion-rate function.$

- power $P\left(G_{n}, U_{n}\right)$ for the transmission of $U_{n}$ bits in a slot when the fading gain is $G_{n}$.

The objective is to minimize the mean delay with constraints on the mean distortion and power. Since, minimizing mean delay is very involved and the mean delay is related to the mean queue length, we try to minimize the mean queue length (observe that since the arrival rate is also a decision variable, minimizing queue lengths, via Little's law, does not guarantee minimizing mean delays). However, we will show later on that such a policy also provides lower mean delay than other policies we are aware of. Thus the controller objective is to obtain an optimal action pair $r=(a, u)$ which minimizes

$$
\limsup _{M \rightarrow \infty} \frac{1}{M} E\left[\sum_{n=0}^{M-1} S_{n}\right]
$$

subject to the constraints

$$
\limsup _{M \rightarrow \infty} \frac{1}{M} E\left[\sum_{n=0}^{M-1} P\left(G_{n}, U_{n}\right)\right] \leq \bar{P}
$$

and

$$
\limsup _{M \rightarrow \infty} \frac{1}{M} E\left[\sum_{n=0}^{M-1} D\left(\frac{A_{n}}{J}\right)\right] \leq \bar{D} .
$$

We show below that this problem may be formulated as an average cost MDP.

In our analysis, channel model (1) itself is not important. Any other channel model with (2) can be used provided the general assumptions (for example assumptions on $P(g, u)$ ) are satisfied. The process $\left\{G_{n}\right\}$ could just represent the channel state.

\section{B. Formulation as an MDP}

Let $\left\{X_{n}, n=0,1, \ldots\right\}$ denote the controlled Markov chain with state space $\mathcal{X}=\mathcal{S} \times \mathcal{G}$, and action space $\mathcal{R}=\mathcal{S} \times \mathcal{A}$. The set of feasible actions in state $x=(s, g)$ is $\mathcal{R}(x):=$ $\{(a, u): u \in\{0,1, \ldots, s\}, a \in \mathcal{A}\}$. Let $\mathcal{K}:=\{(x, r): x \in$ $\mathcal{X}, r \in \mathcal{R}(x)\}$ be the set of all feasible state-action pairs. Let $c$ denote the per stage cost which is a measurable function $c: \mathcal{K} \longrightarrow[0, \infty]$. Let $P(\mathcal{X})$ be the set of all probability measures on $\mathcal{X}$. The conditional probability measure on $\mathcal{X}$ given $\mathcal{K}$ is denoted by $Q(. \mid$.) where

$$
\begin{aligned}
& Q\left(X_{n+1} \in\left\{s^{\prime}\right\} \times G^{\prime} \mid(x, r)\right) \\
& \quad=\delta\left(s^{\prime}=s-u+a\right) \int_{g^{\prime} \in G^{\prime}} d P_{G}\left(g^{\prime} \mid g\right)
\end{aligned}
$$

and $\delta($.$) is the Kronecker delta function.$

Let $\Pi$ be the set of all feasible policies $\pi_{n}$ where

$$
\pi_{n}: \mathcal{K}^{n} \times \mathcal{X} \rightarrow\left\{0,1, \ldots, s_{n}\right\} \times\{0,1, \ldots, \bar{A}\} .
$$


A stationary policy $f \in \Pi$ is a measurable mapping such that

$$
f: \mathcal{X} \rightarrow\{0,1, \ldots, s\} \times\{0,1, \ldots \bar{A}\} .
$$

For a stationary policy $\pi \in \Pi$, we define the buffer cost, the power cost and the distortion cost, respectively, as

and

$$
\begin{aligned}
B^{\pi} & =\limsup _{M \rightarrow \infty} \frac{1}{M} E\left[\sum_{n=0}^{M-1} S_{n}\right] \\
P^{\pi} & =\limsup _{M \rightarrow \infty} \frac{1}{M} E\left[\sum_{n=0}^{M-1} P\left(G_{n}, U_{n}\right)\right]
\end{aligned}
$$

$$
D^{\pi}=\limsup _{M \rightarrow \infty} \frac{1}{M} E\left[\sum_{n=0}^{M-1} D\left(\frac{A_{n}}{J}\right)\right]
$$

when at each stage stationary policy $\pi$ is used. Given $\bar{P}>0, \bar{D}>0$, let $\Pi_{c}$ be the set of feasible policies $\pi \in \Pi$ which satisfy the constraints $P^{\pi} \leq \bar{P}$ and $D^{\pi} \leq \bar{D}$.

The problem can be restated as a constrained optimization problem,

$$
(C P) \text { : Minimize } B^{\pi} \text { subject to } \pi \in \Pi_{c} \text {. }
$$

The problem $(C P)$ can be converted into an unconstrained problem through the Lagrange's method. Let

$$
c(x, r)=s+\beta P(g, u)+\theta D(a)
$$

where $x=(s, g), r=(a, u)$, and $\beta, \theta>0$ are Lagrange multipliers. Also, we assume $J=1$ for simplicity. Define the corresponding Lagrangean functional

$$
J^{\pi}(x)=\limsup _{M \rightarrow \infty} \frac{1}{M} E_{x}^{\pi}\left[\sum_{n=0}^{M-1} c\left(X_{n}, R_{n}\right)\right] .
$$

The following theorem gives sufficient conditions under which an optimal policy for an unconstrained problem is also optimal for the original constrained problem. This is proved in [11] for a single constraint but the same proof works for multiple constraints also.

Theorem 1: Let for some $\beta>0, \theta>0, \pi^{*} \in \Pi$ be the policy that solves the unconstrained problem,

\section{$(U P): \quad$ Minimize $J^{\pi}$ subject to $\pi \in \Pi$.}

If $\pi^{*}$ yields $B^{\pi^{*}}, P^{\pi^{*}}$ and $D^{\pi^{*}}$ as limits with $P^{\pi^{*}}=\bar{P}$ and $D^{\pi^{*}}=$ $\bar{D}$, then the policy $\pi^{*}$ will necessarily solve $C P$.

The posed unconstrained problem $(U P)$ is a standard Markov Decision process with an average cost. In what follows, we solve $U P$ and show that the obtained solution achieves the limits and satisfies the hypothesis of Theorem 1 .

\section{EXISTENCE AND STRUCTURAL RESULTS FOR THE OPTIMAL SOLUTION}

In Section III-A, we show that $(U P)$ has an optimal solution. We also discuss conditions under which this solution is optimal for $(C P)$ also. Section III-B provides some structural results for the optimal policy. Section III-C demonstrates that this optimal policy can be substantially superior to policies which do not optimize the source and channel rates simultaneously.

\section{A. Existence of a Stationary Optimal Policy}

We consider the average cost problem $(U P)$ and define a related $\alpha$-discounted problem.Then we study the average cost problem as a limit of $\alpha$-discounted problems when $\alpha \rightarrow 1$. For $\alpha \in(0,1)$, we define the total expected $\alpha$-discounted cost as

$$
v_{\alpha}(x, \pi)=E_{x}^{\pi}\left[\sum_{n=0}^{\infty} \alpha^{n} c\left(X_{n}, R_{n}\right)\right], \pi \in \Pi, x \in \mathcal{X} .
$$

A policy $\pi^{*}$ satisfying

$$
v_{\alpha}\left(x, \pi^{*}\right)=\min _{\pi \in \Pi} E_{x}^{\pi}\left[\sum_{n=0}^{\infty} \alpha^{n} c\left(X_{n}, R_{n}\right)\right]:=J_{\alpha}(x), x \in \mathcal{X}
$$

is said to be $\alpha$-discount optimal and $J_{\alpha}$ is the $\alpha$-discount optimal cost function. We define corresponding relative gain function as

$$
w_{\alpha}(x)=J_{\alpha}(x)-\inf _{x \in \mathcal{X}} J_{\alpha}(x) \text { for } x \in \mathcal{X} .
$$

The $\alpha$-discount optimality equation ( $\alpha$-DCOE) is

$$
v_{\alpha}(x)=\min _{r \in \mathcal{R}(x)}\left[c(x, r)+\alpha \int_{x^{\prime} \in \mathcal{X}} v_{\alpha}\left(x^{\prime}\right) d Q\left(x^{\prime} \mid(x, r)\right)\right]
$$

and $J_{\alpha}(x)$ is the minimal solution to the $\alpha$-DCOE. We will assume that $J^{*}:=\inf _{x \in \mathcal{X}} \inf _{\pi \in \Pi} J^{\pi}(x)<\infty$. This assumption should hold in most practical problems because otherwise the cost is infinite for any choice of the policy and thus any policy is optimal, anyway.

Consider the following set $(\mathbf{S})$ of conditions [13]:

$\mathrm{S} 1) \mathcal{R}(x)$, the set of feasible actions in state $x$ is a compact subset of $\mathcal{R}$ for all $x \in \mathcal{X}$.

S2) $Q\left(x^{\prime} \in B^{\prime} \mid x,.\right): \mathcal{R}(x) \longrightarrow P(\mathcal{X})$ is continuous with respect to set-wise convergence on $P(\mathcal{X})$ for $x \in \mathcal{X}, B^{\prime} \in \mathcal{G}(\mathcal{X})$. S3) $c(x,):. \mathcal{R}(x) \longrightarrow[0, \infty]$ is lower semicontinuous for all $x \in \mathcal{X}$.

It is easy to verify these conditions for our system because of the discreteness of the buffer state and action spaces. The following lemma [13] gives the conditions for existence of stationary discounted optimal policies.

Lemma 1: Let $\alpha \in(0,1)$. Under the condition $(\mathbf{S})$ there exists a discount optimal stationary policy $\pi_{\alpha}$ which satisfies the $\alpha$-DCOE.

The next theorem gives the conditions for the existence of average optimal policies which can be obtained as a limit of discounted optimal policies $\pi_{\alpha}$. Let $\nu_{g, \bar{G}}$ be the number of jumps required for Markov chain $\left\{G_{n}\right\}$ to visit the state $\bar{G}$ for the first time when it starts in state $g$. Motivated by [13] we have the following theorem.

Theorem 2: There exists a policy $\pi^{*}$ which is average cost optimal for $(U P)$ and $J^{\pi^{*}}(x)=J^{*}$ for all $x$. Also, $\pi^{*}$ is limit discount optimal in the sense that for any $x \in \mathcal{X}$ and given any sequence of discount factors converging to one, there exists some subsequence $\alpha_{n} \rightarrow 1$ such that

$$
\pi^{*}(x)=\lim _{n \rightarrow \infty} \pi_{\alpha_{n}}(x)
$$

Furthermore

$$
J^{*}=\lim _{\alpha \rightarrow 1}(1-\alpha) \inf _{x \in \mathcal{X}} J_{\alpha}(x) .
$$

Proof: In [13] it is shown that if

$$
\sup _{\alpha<1} w_{\alpha}(x)<\infty \text { for all } x \in \mathcal{X}
$$

and conditions (S) are satisfied then the conclusions of this theorem hold. We have already stated that conditions $(\mathbf{S})$ hold under our assumptions. To prove $\sup _{\alpha<1} w_{\alpha}(x)<\infty$ for all $x \in \mathcal{X}$, first we prove that $J_{\alpha}(x)<\infty$ for all $x \in \mathcal{X}$ and $\alpha \in(0,1)$. 
For a state $x=(s, g)$, let $\pi$ be the policy such that $u=s, a=\bar{A}$. Let $c_{n}$ be the cost per stage. Then for $n \geq 1$, the cost incurred is upper bounded by $\bar{c}=\bar{A}+\beta P(\bar{g}, \bar{A})+\theta D(0)<\infty$. Thus

$$
\begin{aligned}
J_{\alpha}(x) & \leq s+\beta P(g, s)+\theta D(\bar{A})+E_{x}^{\pi}\left[\sum_{n=1}^{\infty} \alpha^{n} \bar{c}\right] \\
& =s+\beta P(g, s)+\theta D(\bar{A})+\alpha \frac{\bar{c}}{(1-\alpha)}<\infty .
\end{aligned}
$$

Now we will show that $\sup _{\alpha<1} w_{\alpha}(x)<\infty$ for all $x \in \mathcal{X}$. We will prove in Propositions 1 and 2 below that $J_{\alpha}(s, g)$ is increasing in $s$ and decreasing in $g$. Therefore, $\arg \inf _{x \in \mathcal{X}} J_{\alpha}(x)=J_{\alpha}\left(x_{0}\right)$ where $x_{0}=(0, \bar{G})$. Let $r_{1}=\left(0, a_{g}\right)$ be the optimal policy for state $(0, g)$. Then we have the value function

$$
J_{\alpha}(0, g)=\theta D\left(a_{g}\right)+\alpha \int_{g^{\prime} \in \mathcal{G}} J_{\alpha}\left(a_{g}, g^{\prime}\right) d P_{G}\left(g^{\prime} \mid g\right)
$$

for state $(0, g)$. For any $x \in \mathcal{X}$ choose a feasible policy $r(x)=\left(s, a_{g}\right)$ then

$$
J_{\alpha}(s, g) \leq s+\beta P(g, s)+\theta D\left(a_{g}\right)+\alpha \int_{g^{\prime} \in \mathcal{G}} J_{\alpha}\left(a_{g}, g^{\prime}\right) d P_{G}\left(g^{\prime} \mid g\right) .
$$

From (14) and (15), we have for all $(s, g) \in \mathcal{X}$

$$
J_{\alpha}(s, g) \leq s+\beta P(g, s)+J_{\alpha}(0, g)
$$

Therefore

$$
w_{\alpha}(s, g) \leq s+\beta P(g, s)+J_{\alpha}(0, g)-J_{\alpha}(0, \bar{G})
$$

and hence it is sufficient to get an upper bound on $\sup _{\alpha<1} J_{\alpha}(0, g)$.

Consider the state $(0, g)$. Let us use a policy under this state, $r=$ $(0,0)$ till there is a transition to state $g=\bar{G}$. The cost incurred per slot by this policy can be upper bounded by $\bar{c}=\theta D(0)$. Therefore, starting from state $(0, g)$, if $c_{k}$ is the cost of $k$ th stage of the value iteration

$$
J_{\alpha}(0, g) \leq E\left[\sum_{k=0}^{\infty} \alpha^{k} c_{k}\right] \leq E\left[\sum_{k=0}^{\nu_{g}, \bar{G}} \bar{c}\right]+J_{\alpha}\left(x_{0}\right)
$$

Therefore, from (17) it follows that

$$
w_{\alpha}(s, g) \leq s+\beta P(g, s)+\bar{c} E\left[\nu_{g, \bar{G}}\right] .
$$

Since the bound is independent of $\alpha$, we obtain

$$
\sup _{\alpha<1} w_{\alpha}(x) \leq s+\beta P(g, s)+\bar{c} E\left[\nu_{g, \bar{G}}\right]<\infty \text { for all } x \in \mathcal{X}
$$

Although we have limited ourselves to stationary deterministic policies, the above theorem in fact guarantees existence of an optimal stationary deterministic policy in the class of all randomized (not necessarily stationary) policies.

Under the optimal policy obtained in Theorem $2, J^{\pi^{*}}$ is finite. Then by Theorem $E 10$ of [6, p. 188], there exists an invariant distribution for the Markov chain $\left\{X_{n}\right\}$ under the optimal policy $\pi^{*}$. Also, by Theorem $E 11$ of [6, p. 188], for policy $\pi^{*}, B^{\pi^{*}}, P^{\pi^{*}}$ and $D^{\pi^{*}}$ exist as limits. If under $\pi^{*}$ the Markov chain is irreducible, these limits are independent of the initial conditions. If the Markov chain under the $\pi^{*}$ has a single communicating class (but the chain is not necessarily irreducible), then also, after modifying the optimal policy initially so that in one step one enters that communicating class, the limits $\pi^{*}, B^{\pi^{*}}, P^{\pi^{*}}$ and $D^{\pi^{*}}$ can be made independent of the initial state. Furthermore, as $\beta$ increases $P^{\pi^{*}}$ decreases and $D^{\pi^{*}}$ increases and as $\theta$ decreases $D^{\pi^{*}}$ increases and $P^{\pi^{*}}$ decreases. Thus by choosing $\theta, \beta$ appropriately, we can ensure that $P^{\pi^{*}}=\bar{P}$ and $D^{\pi^{*}}=\bar{D}$. Then all the conditions of Theorem 1 are satisfied.

\section{B. Structural Results}

In this section, we obtain some structural results on the optimal cost and policies. Some of these have been used in Section III-A. We will first obtain the results for $n$-stage $\alpha$-value iteration function $J_{n}(x)$ when the initial state is $x$. Then using the fact (to be proved below), that for any $\alpha, 0<\alpha<1, J_{\alpha}(x)=\lim _{n \rightarrow \infty} J_{n}(x)$, we will obtain the results for $J_{\alpha}(x)$. Finally the results will be extended to $J^{*}$.

It is proved in [6] that if the conditions (A)

A1) The one-stage cost $c$ is lower semicontinuous, nonnegative, and inf-compact on $\mathcal{K}$, i.e., the set $\{r \in \mathcal{R}(x): c(x, r) \leq t\}$ is compact for every $x \in \mathcal{X}$ and $t \in \Re$.

A2) $\mathrm{Q}$ is strongly continuous, i.e., $Q(B \mid$.) is continuous on $\mathcal{K}$ for every set $B \in \mathcal{G}(\mathcal{X})$.

A3) There exists a policy $\pi$ such that $J_{\alpha}^{\pi}(x)<\infty$ for each $x \in \mathcal{X}$. are satisfied, then $J_{n}(x)$ converges to $J_{\alpha}(x)$ which satisfies $\alpha$-DCOE.

By the discreteness of the buffer and action spaces, and by the assumptions made in Section II-A, the conditions A1 and A2 can be easily verified. Condition A3 has already been verified in Section III.

Now we obtain the structural results for the $\alpha$-value function and $\alpha$-discount optimal policy for each $\alpha \in(0,1)$. It follows from Theorem 2 that the same will hold for average cost function and optimal policies also.

Proposition 1: $J_{\alpha}(s, g)$ is non-decreasing in $s$ for all $g \in \mathcal{G}$.

Proof: This can be proved by induction on the value iteration algorithm

$$
\begin{aligned}
J_{n+1}(s, g)=\min _{(u, a) \in \mathcal{R}(x)}\{s & +\beta P(g, u) \theta D(a) \\
& \left.+\alpha \int_{g^{\prime} \in \mathcal{G}} J_{n}\left(s-u+a, g^{\prime}\right) d P_{G}\left(g^{\prime} \mid g\right)\right\} .
\end{aligned}
$$

For $n=0$ we have $J_{0}(s, g)=$ constant (say $C$ ). Therefore, $J_{0}(s, g)=$ is nondecreasing. Assume $J_{n}(s, g)$ is nondecreasing in $s$ for each $g$. Fix $g$. Let $s_{1}$ and $s_{2}$ be the two states such that $s_{2}>s_{1}$. Let $\left(u_{1}, a_{1}\right)$ and $\left(u_{2}, a_{2}\right)$ be the optimal policies corresponding to states $\left(s_{1}, g\right)$ and $\left(s_{2}, g\right)$ for the $n+1$-stage problem. Thus

$$
\begin{aligned}
J_{n+1}\left(s_{2}, g\right)=s_{2}+\beta & P\left(g, u_{2}\right)+\theta D\left(a_{2}\right) \\
& +\alpha \int_{g^{\prime} \in \mathcal{G}} J_{n}\left(s_{2}-u_{2}+a_{2}, g^{\prime}\right) d P_{G}\left(g^{\prime} \mid g\right) .
\end{aligned}
$$

We note that $r_{2}=\left(a_{2}, u_{2}\right)$, is a feasible policy for state $\left(s_{2}, g\right)$. We apply policy $r_{2}=\left(a_{2}, u_{2}\right)$ for the state $s_{1}$. When $u_{2}<s_{1}$, we have

$$
\begin{aligned}
J_{n+1}\left(s_{1}, g\right) \leq & s_{1}+\beta P\left(g, u_{2}\right)+\theta D\left(a_{2}\right) \\
& +\alpha \int_{g^{\prime} \in \mathcal{G}} J_{n}\left(s_{1}-u_{2}+a_{2}, g^{\prime}\right) d P_{G}\left(g^{\prime} \mid g\right) \\
\leq & s_{2}+\beta P\left(g, u_{2}\right)+\theta D\left(a_{2}\right) \\
& +\alpha \int_{g^{\prime} \in \mathcal{G}} J_{n}\left(s_{2}-u_{2}+a_{2}, g^{\prime}\right) d P_{G}\left(g^{\prime} \mid g\right) . \\
= & J_{n+1}\left(s_{2}, g\right)
\end{aligned}
$$

where the first inequality follows from the optimality of policy $\left(a_{1}, u_{1}\right)$ for $\left(s_{1}, g\right)$ and the second one follows since $s_{2}>s_{1}$ and $J_{n}(., g)$ is nondecreasing. When $u_{2}>s_{1}$ apply $r_{2}^{\prime}=\left(s_{1}, a_{2}\right)$ to the state $s_{1}$. 
Then

$$
\begin{aligned}
J_{n+1}\left(s_{1}, g\right) \leq & s_{1}+\beta P\left(g, s_{1}\right)+\theta D\left(a_{2}\right) \\
& +\alpha \int_{g^{\prime} \in \mathcal{G}} J_{n}\left(a_{2}, g^{\prime}\right) d P_{G}\left(g^{\prime} \mid g\right) \\
\leq & s_{2}+\beta P\left(g, u_{2}\right)+\theta D\left(a_{2}\right) \\
& +\alpha \int_{g^{\prime} \in \mathcal{G}} J_{n}\left(s_{2}-u_{2}+a_{2}, g^{\prime}\right) d P_{G}\left(g^{\prime} \mid g\right) \\
= & J_{n+1}\left(s_{2}, g\right)
\end{aligned}
$$

where the above inequalities follow from the optimality of policies and non-decreasing nature of $P(g,$.$) and J_{n}(., g)$. Hence we have proved that $J_{n+1}(., g)$ is nondecreasing. Since $J_{\alpha}()=.\lim _{n \rightarrow \infty} J_{n}($.$) , we$ obtain the result for $J_{\alpha}$.

Proposition 2: $J_{\alpha}(s, g)$ is non-increasing in $g$ for all $s \in \mathcal{S}$.

Proof: As in the above proof, we prove the proposition by induction on the value iteration algorithm.

Since $J_{0}(s, g)=$ constant, it is non-increasing in $g$. Assume $J_{n}(s, g)$ is non-increasing in $g$ for each $s$. Fix $s$. Let $g_{1}$ and $g_{2}$ be two states with $g_{2}>g_{1}$. Let $\left(a_{1}, u_{1}\right)$ and $\left(a_{2}, u_{2}\right)$ be the optimal policies corresponding to $\left(s, g_{1}\right)$ and $\left(s, g_{2}\right)$ respectively for the $n+1$-stage problem. Thus,

$$
\begin{aligned}
& J_{n+1}\left(s, g_{2}\right)=s+\beta P\left(g_{2}, u_{2}\right)+\theta D\left(a_{2}\right) \\
& +\alpha \int_{g^{\prime} \in \mathcal{G}} J_{n}\left(s-u_{2}+a_{2}, g^{\prime}\right) d P_{G}\left(g^{\prime} \mid g_{2}\right) .
\end{aligned}
$$

We apply policy $r_{1}=\left(a_{1}, u_{1}\right)$ for the state $g_{2}$. Hence we have $n+$ 1 -stage cost for $\left(s, g_{2}\right)$ as

$$
\begin{aligned}
J_{n+1}\left(s, g_{2}\right) \leq & s+\beta P\left(g_{2}, u_{1}\right)+\theta D\left(a_{1}\right) \\
& +\alpha \int_{g^{\prime} \in \mathcal{G}} J_{n}\left(s-u_{1}+a_{1}, g^{\prime}\right) d P_{G}\left(g^{\prime} \mid g_{2}\right) \\
\leq & s+\beta P\left(g_{1}, u_{1}\right)+\theta D\left(a_{1}\right) \\
& +\alpha \int_{g^{\prime} \in \mathcal{G}} J_{n}\left(s-u_{1}+a_{1}, g^{\prime}\right) d P_{G}\left(g^{\prime} \mid g_{2}\right) .
\end{aligned}
$$

Now we use the fact that the fading process $\left\{G_{n}\right\}$ is stochastically monotone and $J_{n}($.$) is non-increasing in g$. Then for all $g_{1}, g_{2} \in \mathcal{G}, g_{1}<g_{2}$

$$
\int_{g^{\prime} \in \mathcal{G}} J_{n}\left(s, g^{\prime}\right) d P_{G}\left(g^{\prime} \mid g_{2}\right) \leq \int_{g^{\prime} \in \mathcal{G}} J_{n}\left(s, g^{\prime}\right) d P_{G}\left(g^{\prime} \mid g_{1}\right) .
$$

Using the above inequality in (19), we get

$$
\begin{aligned}
J_{n+1}\left(s, g_{2}\right) \leq & s+\beta P\left(g_{1}, u_{1}\right)+\theta D\left(a_{1}\right) \\
& +\alpha \int_{g^{\prime} \in \mathcal{G}} J_{n}\left(s-u_{1}+a_{1}, g^{\prime}\right) d P_{G}\left(g^{\prime} \mid g_{1}\right) \\
= & J_{n+1}\left(s, g_{1}\right) .
\end{aligned}
$$

Thus $J_{n}(s, g)$ is non-increasing in $g$.

Proposition 3: $J_{\alpha}(s+1, g)-J_{\alpha}(s, g)$ is non-decreasing in $s$ for all $g \in \mathcal{G}$.

Proof: Refer to Appendix I.

Proposition 4: Fix $g$. Let $(a(s), u(s))$ be an optimal policy for buffer state $s$. Define $r(s)=u(s)-a(s)$. Then $r(s)$ is nondecreasing in $s$.

Proof: This will be proved by contradiction. Let $s_{1}$ and $s_{2}$ be the two states such that $s_{1}>s_{2}$. Let the corresponding optimal policies be $\left(a_{1}, u_{1}\right)$ and $\left(a_{2}, u_{2}\right)$. Let $r_{i}=u_{i}-a_{i}, i=1,2$. Assume $r_{1}<r_{2}$.
We have the cost function

$$
\begin{aligned}
& J_{\alpha}(s, g)=\min _{(u, a) \in \mathcal{R}(x)}\left\{s+\beta P(g, u)+\theta D(a)+V_{\alpha}(s-r)\right\} \\
& \text { where } \\
&\left.V_{\alpha}(s)=\alpha \int_{g^{\prime} \in \mathcal{G}} J_{\alpha}\left(s, g^{\prime}\right) d P_{G}\left(g^{\prime} \mid g\right)\right\}=\alpha E_{g^{\prime} \mid g}\left[J_{\alpha}\left(s, g^{\prime}\right)\right] .
\end{aligned}
$$

Thus

$$
\begin{aligned}
J_{\alpha}\left(s_{1}, g\right) & =s_{1}+\beta P\left(g, u_{1}\right)+\theta D\left(a_{1}\right)+V_{\alpha}\left(s_{1}-r_{1}\right) \\
& \leq s_{1}+\beta P\left(g, u_{1}\right)+\theta D\left(a_{2}\right)+V_{\alpha}\left(s_{1}-r_{2}\right) .
\end{aligned}
$$

Also

$$
\begin{aligned}
J_{\alpha}\left(s_{2}, g\right) & =s_{2}+\beta P\left(g, u_{2}\right)+\theta D\left(a_{2}\right)+V_{\alpha}\left(s_{2}-r_{2}\right) \\
& \leq s_{2}+\beta P\left(g, u_{2}\right)+\theta D\left(a_{1}\right)+V_{\alpha}\left(s_{2}-r_{1}\right) .
\end{aligned}
$$

Adding (20) and (21) we get

$$
V_{\alpha}\left(s_{1}-r_{1}\right)-V_{\alpha}\left(s_{1}-r_{2}\right) \leq V_{\alpha}\left(s_{2}-r_{1}\right)-V_{\alpha}\left(s_{2}-r_{2}\right) .
$$

From Proposition 3 this leads to contradiction.

\section{Example}

In this section we obtain the optimal average cost solution for an example and then compare it to other known suboptimal solutions. The channel fading has Rayleigh distribution. The fading values are discretized into an 8-state Markov model. Number of channel uses per slot is $N=10$. The source is independent and identically distributed (i.i.d. ) $Z \sim \mathcal{N}(0,1)$ and the channel noise is AWGN with $W \sim \mathcal{N}(0,1)$. Jake's model was used to generate the fading process with eight multipath components and a Doppler frequency of $5 \mathrm{~Hz}$. This enabled to simulate an isotropic scattering environment with high autocorrelation. We computed the optimal policies through the value iteration algorithm. We choose Lagrange multipliers $\beta$ and $\theta$ such that under the optimal policy the average policies and distortions converged to the required constraints. Thus they satisfy the requirements of Theorem 1 . We compare the costs incurred by our policy with the following two suboptimal policies for a fixed mean distortion of $1.5 \%$

1) Policy A. Source Coder (SC) Uses Only the Buffer State Information and Channel Coder (CC) Uses Only the Channel State Information: In this case, the $\mathrm{CC}$ tries to maximize the transmission rate (channel capacity) for the given average power constraint. This scenario corresponds to the case when the physical layer and higher layers have no coupling. This provides the well known water filling power control. The power policy obeys

$$
P(g)=\left[\frac{1}{\lambda}-\frac{1}{g^{2}}\right]^{+}
$$

where $\lambda$ is the solution of the constraint equation

$$
\int_{g \geq \sqrt{\lambda}}\left[\frac{1}{\lambda}-\frac{1}{g^{2}}\right] d \mu_{G}(g)=\bar{P}
$$

and the corresponding $u=\left\lfloor N \log \left(1+\frac{P(g) g^{2}}{\sigma_{w}^{2}}\right)\right\rfloor$.

The SC adapts the source coding rate based on the buffer content according to the optimal solution of

$$
\begin{aligned}
& J_{n+1}(s, g) \\
& \quad=\min _{a}\left\{s+\theta D(a)+\int_{g^{\prime} \in \mathcal{G}} J_{n}\left(s-u+a, g^{\prime}\right) d P_{G}\left(g^{\prime} \mid g\right)\right\} .
\end{aligned}
$$




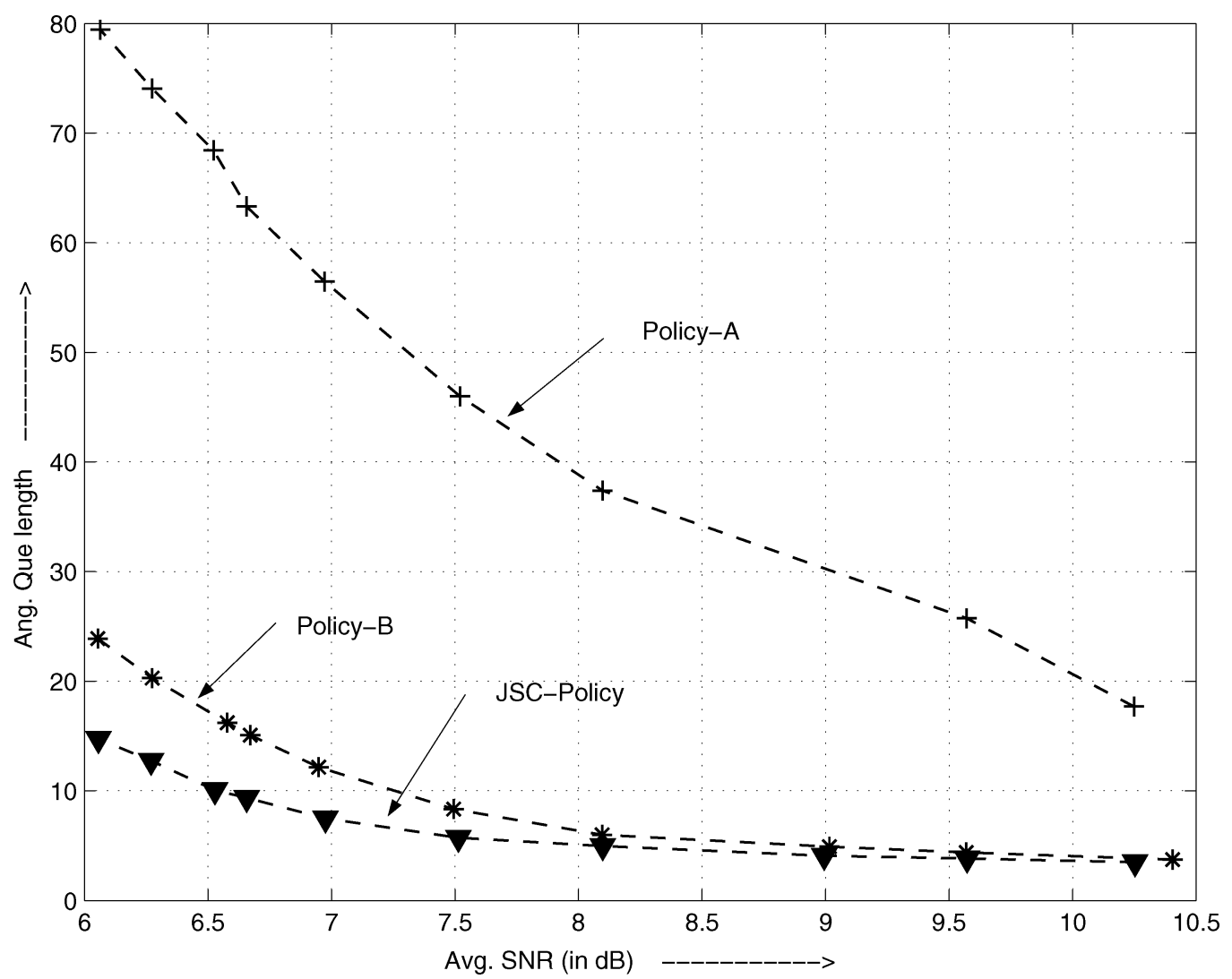

Fig. 1. Average queue length versus average SNR.

2) Policy B. SC With No State Information and CC With Complete State Information: This case is studied in [4] and [1]. Now the SC generates an i.i.d. sequence of bits satisfying the average distortion constraint with equality. The CC has both the channel and the buffer state information. The CC controls the transmission power obeying the delay and power constraints. For the given constraints we computed the optimal policy obtained in [4] and [1].

Fig. 1 provides the comparison between the power-workload tradeoff curves of the JSC policy (the optimal policy obtained here), policy A and policy B for a given mean distortion (= 1.5\%). The JSC (optimal) policy achieves much lower mean queue length than both policy A and policy B at low signal-to-noise ration (SNR). As the SNR increases, the difference in performance of the three policies goes down. This is intuitively satisfying because as power increases, the entire buffer can be served and hence all the three policies must provide similar performance. We also observe that policy-B performs much better than policy-A. For example, at $6 \mathrm{~dB}$, the mean queue length of policy-A is 80 , of policy-B is 25 and of JSC is 15 .

Fig. 2 plots the mean delays of the three policies. We observe that the mean delays of the three policies behave in the same way as the mean queue lengths. This justifies minimizing mean queue lengths even though we may be actually interested in minimizing the mean delay.

\section{Single USER, MIMO SySTEM}

In previous sections, we have considered a single user communicating over a fading channel with a single transmit and receive antenna single-input-single-output (SISO). Here, we consider a slotted system with point-to-point communication with $N_{t}$ transmit antennas and $N_{r}$ receive antennas over a narrowband flat-fading wireless channel. It is well known that the potential gain in achievable rates of MIMO systems is rather large compared to single antenna systems under "spa- tially uncorrelated fading" assumptions. When the scattering environment is rich enough to provide independent transmission paths from each transmit antenna to receive antenna with perfect estimates of the channel gains being available at receiver, the capacity scales linearly with $\min \left(N_{t}, N_{r}\right)$ relative to a SISO case [12]. To make use of the spectral efficiency of MIMO systems, we will extend our previous work to MIMO case. We show that given mean power and mean distortion, the mean queue length can be significantly reduced compared to SISO case.

Let $\mathbf{G}_{n}$ be the fading matrix in slot $n$. The elements of $\mathbf{G}_{n}, g_{i, j}(i=$ $\left.1, \ldots, N_{r}, j=1, \ldots, N_{t}\right)$ are assumed to be identically distributed and belong to the space $\mathcal{G}$ so that $\mathbf{G}_{n} \in \mathcal{G}^{N_{r} \times N_{t}} . \mathbf{G}_{n}$ evolves as an ergodic Markov chain with a stationary distribution and transition density $P_{\mathrm{G}}$. We assume that the instantaneous channel coefficient matrix $\mathbf{G}_{n}$ and buffer state information are available both at the transmitter and the receiver in each slot. There are $N$ channel uses in a slot. The channel fading gain remains constant during a slot. With appropriate sampling, the discrete representation of the channel in the $n^{t h}$ slot is

$$
\mathbf{Y}_{n}=\mathbf{G}_{n} \mathbf{Z}_{n}+\mathbf{W}_{n}, \quad n=0,1, \ldots
$$

where $\mathbf{Y}_{n}$ is the $N_{r} \times 1$ received signal vector and $\mathbf{Z}_{n}$ is the $N_{t} \times 1$ transmitted signal vector. $\mathbf{W}_{n}$ is the AWGN noise with the covariance matrix $E\left[\mathbf{W}_{n} \cdot \mathbf{W}_{n}^{H}\right]=\sigma_{w}^{2} \cdot \mathbf{I}_{N_{r}}$.

We know that [12] a MIMO channel can be viewed as a combination of $L$ independent SISO channels each having a channel transfer function $\lambda_{i}, i=1, \ldots, L$, where $L$ is the rank of the channel matrix $\mathbf{G}_{n},\left(L \leq \min \left(N_{r}, N_{t}\right)\right)$ and $\lambda_{i}$ is the eigenvalue of the matrix $\mathbf{G}_{n} \mathbf{G}_{n}^{H}$. Let $P_{i}$ be the power pumped into the $i$ th spatial channel. Then the instantaneous capacity of the corresponding channel is given by

$$
C_{i}=\frac{1}{2} \log _{2}\left(1+\frac{P_{i} \lambda_{i}}{\sigma_{w}^{2}}\right), i=1, \ldots, L .
$$




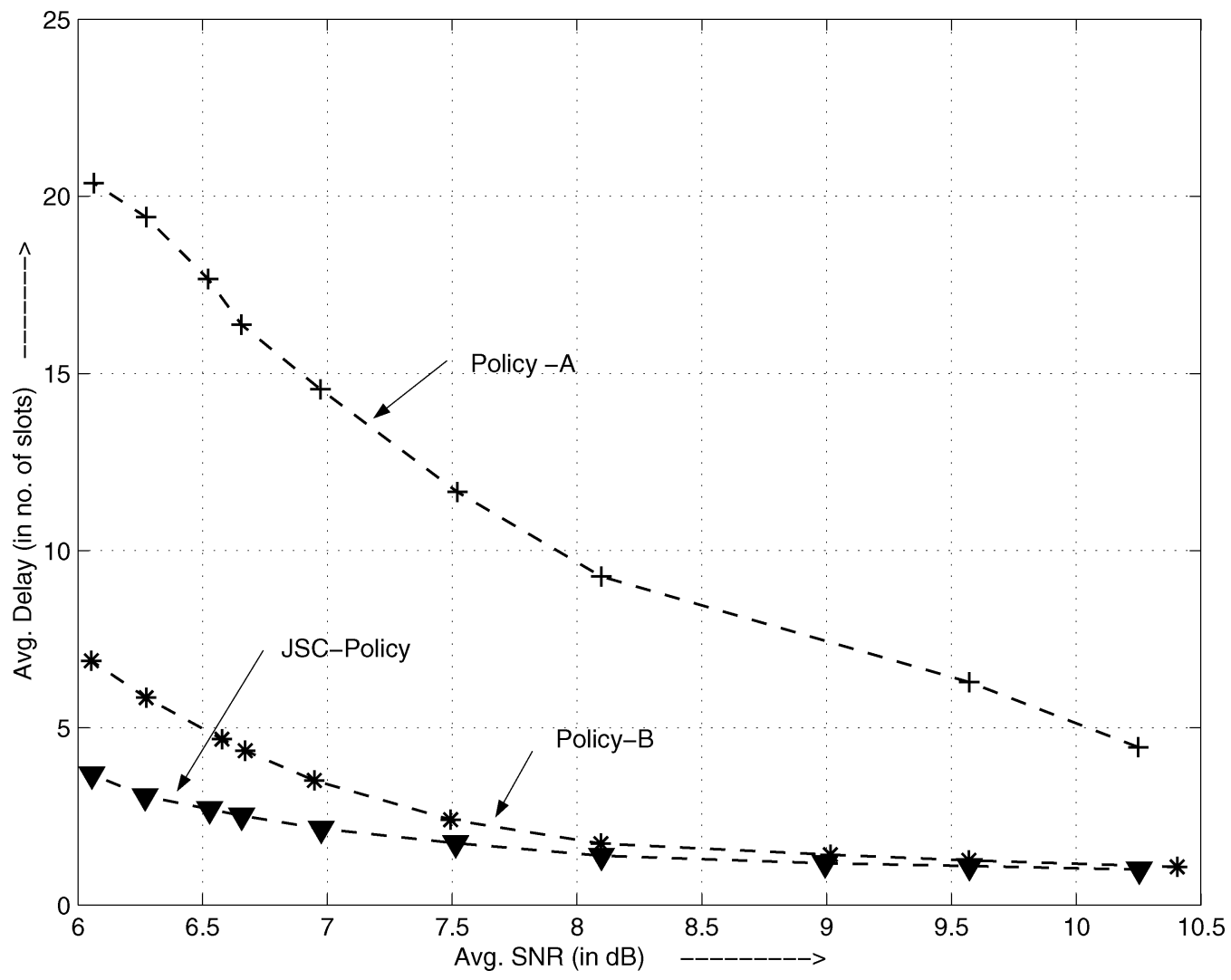

Fig. 2. Average delay versus average SNR.

Hence, if $u_{i}$ is the number of bits per slot transmitted in $i$ th spatial mode then, the power expended is

$$
P_{i}=\frac{\sigma_{w}^{2}}{\lambda_{i}}\left(e^{k u_{i}}-1\right)
$$

where $k=\frac{2 \ln (2)}{N}$.

We define the state of the system in the $n^{t h}$ slot as $X_{n}:=\left(S_{n}, \mathbf{G}_{\mathbf{n}}\right)$. At the $n$th decision epoch, the transmitter decides upon the amount of data bits $A_{n}$ to be placed in the buffer and the amount of data bits $U_{n}$ to be removed from the buffer and distributed optimally across the $L$ spatial subchannels for the transmission. As it was with the SISO case, the above model also satisfies the conditions ( given in Lemma 1 and Theorem 2) required for the existence of discount optimal and average optimal stationary policies. Therefore, the value function can be obtained as a solution to the average cost dynamic programming equation

$$
\begin{aligned}
J_{n+1}(s, \mathbf{G})=\min _{a, u}\{s+\theta D(a)+\beta P(\mathbf{G}, u) \\
\left.+\int_{\mathbf{G}^{\prime} \in \mathcal{G} N_{r} \times N_{t}} J_{n}\left(s-u+a, \mathbf{G}^{\prime}\right) d P_{G}\left(\mathbf{G}^{\prime} \mid \mathbf{G}\right)\right\}
\end{aligned}
$$

where

$$
P(\mathbf{G}, u)=\min _{\mathbf{0} \leq \mathbf{u}: \sum_{i} u_{i}=u} \sum_{i=1}^{L} P_{i} .
$$

We will show below that, for the given total transmission rate, the optimal power allocation policy obeys the water-filling rule.

We can solve the above constrained problem (26) by defining a Lagrangean

$$
P_{\mu}(u, \mathbf{G})=\min _{\mathbf{u} \geq 0} \sum_{i=1}^{L}\left(P_{i}-\mu u_{i}\right)
$$

$$
=\min _{\mathbf{u} \geq 0} \sum_{i=1}^{L}\left(\frac{\sigma_{w}^{2}}{\lambda_{i}}\left(e^{k u_{i}}-1\right)-\mu u_{i}\right)
$$

where $\mu$ is the Lagrange multiplier. Setting $\frac{\partial P_{\mu}}{\partial u_{i}}=0$, and solving for $u_{i}$, we have

$$
u_{i}=\frac{1}{k} \ln \left(\frac{\mu}{k \sigma_{w}^{2}} \lambda_{i}\right) \text { for all } i
$$

where $\mu$ is obtained from $u=\sum_{i=1}^{L} u_{i}$.

Thus we obtain,

$$
u_{i}=\min \left[\left[\frac{1}{k L} \ln \left(e^{k u} \prod_{j=1}^{L} \frac{\lambda_{i}}{\lambda_{j}}\right)\right]^{+}, u\right] .
$$

Hence, the $i$ th channel incurs a power cost of

$$
P_{i}=\min \left[\left[\mu^{\prime}-\frac{\sigma_{w}^{2}}{\lambda_{i}}\right]^{+}, \frac{\sigma_{w}^{2}}{\lambda_{i}}\left(e^{k u}-1\right)\right]
$$

where $\mu^{\prime}=\sigma_{w}^{2}\left[\left(\frac{e^{k u}}{\prod_{j=1}^{L} \lambda_{j}}\right)^{\frac{1}{L}}-\frac{1}{\lambda_{i}}\right]^{+}$. This obeys the well known water-filling rule in space. The total power expended in slot $n$ for a given $u$ is

$$
P(\mathbf{G}, u)=\sum_{i=1}^{L}\left[\mu^{\prime}-\frac{\sigma_{w}^{2}}{\lambda_{i}}\right]^{+}
$$

We can see that more bits are allotted to the subchannel with the larger eigenvalue. 


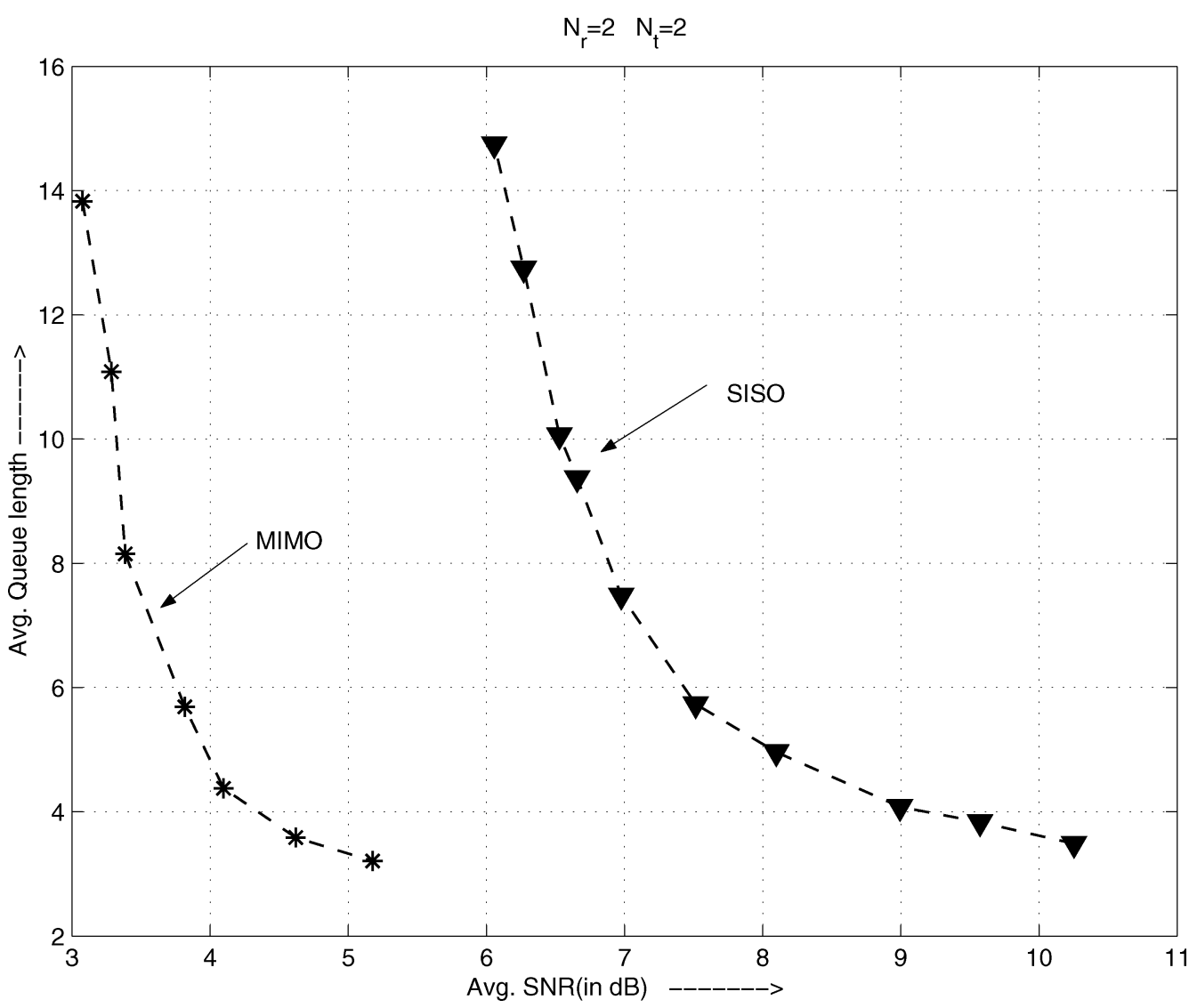

Fig. 3. Average buffer length versus average power for JSC policies of SISO and MIMO cases with.

Next, we compare the performance of the MIMO system with the SISO system of Section III-C. The MIMO system has $N_{t}=N_{r}=2$ and the channel fading process is i.i.d. across space. Fig. 3 gives the plot of average queue length vs power curves of both the systems.

We can see from the plot that for a given mean queue length, the MIMO system achieves nearly $3-4 \mathrm{~dB}$ gain in average power.

\section{Multiuser CASE}

We consider a slotted multi-user uplink system over a narrowband fading wireless channel with $M$ users communicating to a base station receiver. For each user, data to be transmitted, arrives from a higher layer and this data is subjected to a variable rate compression depending on queue length, power and distortion requirements. This compressed data is placed into the transmission buffer. We assume that the buffer state information of all the users is available both at each transmitters and the receiver. We also assume perfect CSIT and perfect CSIR, i.e., all the transmitters and the receiver know current state of the channels of every user. The channel is assumed to be block fading, so that the fading gain remains constant throughout a slot. We will assume that each user has a single transmit antenna and the base station also has a single receive antenna. Extension to multiple transmit and receive antennas can be done in the same way as for a single user.

The discrete representation of the channel during $n^{\text {th }}$ slot is

$$
Y_{n}=\sum_{i=1}^{M} G_{n}(i) Z_{n}(i)+W_{n}, \quad n=0,1, \ldots
$$

where $Y_{n}$ is the output of the channel, $Z_{n}(i)$ is the transmitted symbol of $i$ th user in $n^{\text {th }}$ slot and $G_{n}(i)$ is the fading gain process of $i$ th user and $G_{n}(i) \in \mathcal{G}:=\left[\bar{g}^{2}, \bar{G}^{2}\right]$. Take $H_{n}(i)=\left|G_{n}(i)\right|^{2}$ and hence,
$H_{n}(i) \in \mathcal{H}:=[\bar{h}, \bar{H}]$ for all $i$. Henceforth, without loss of generality we will use fading power $(h)$ instead of fading gain. $W_{n}$ is additive white Gaussian Noise process with variance $\sigma_{n}^{2}=\sigma^{2}$. Denote by $\mathcal{M}:=\{1, \ldots, M\}$ the set of transmitters. We assume that the fading processes of all users are stationary ergodic, stochastically monotone Markov chains. User $i$ is also subject to a transmitter power constraint $\bar{P}_{i}$ and a distortion constraint $\bar{D}_{i}$.

Let $\mathbf{H}_{n}=\left[H_{n}(1), \ldots, H_{n}(M)\right]$ be a random vector representing the joint fading state during $n^{\text {th }}$ channel block. Similarly, let $\overline{\mathbf{P}}=$ $\left[\bar{P}_{1}, \ldots, \bar{P}_{M}\right]$ be a power vector and $\overline{\mathbf{D}}=\left[\bar{D}_{1}, \ldots, \bar{D}_{M}\right]$ be a distortion vector.

Let $S_{n}(i)$ be the amount of data in the buffer of user $i$ at the beginning of $n^{\text {th }}$ slot and $S_{n}(i) \in \mathcal{S}$ where $\mathcal{S}=\{0,1, \ldots\}$ and let $\mathbf{S}_{n}=\left[S_{n}(1), \ldots, S_{n}(M)\right]$. We define the state vector of the system at the $n^{\text {th }}$ slot as $\mathbf{X}_{n}:=\left(\mathbf{S}_{n}, \mathbf{H}_{n}\right)$. At the $n$th decision epoch, the $i$ th transmitter places $A_{n}(i)$ bits in the buffer and removes $U_{n}(i)$ bits from the buffer for transmission. The evolution of the buffer state process for all $i \in \mathcal{M}$, is given by

$$
S_{n+1}(i)=S_{n}(i)+A_{n}(i)-U_{n}(i), n=0,1, \ldots
$$

where $U_{n}(i) \in\left\{0,1, \ldots, S_{n}(i)\right\}$ and $A_{n}(i) \in\{0,1 \ldots, \bar{A}\}, \bar{A}<\infty$ In this section we obtain a jointly source and channel rate optimal policy for multiuser case. In Section V-A, we recall some results which will be used later. In Section V-B, we formulate the optimization problem as an MDP. In Section V-C, we provide structural results for the optimal policy. The optimal policy is computed via value iteration. For multiuser case, it is very computationally intensive. Therefore, in Section V-D, we obtain an explicit suboptimal policy which can be justified intuitively and is easy to compute. We compare it with the optimal policy for a particular example. 


\section{A. Preliminaries}

Let the receiver noise power be $\sigma^{2}$. Define

$$
\mathbf{R}(\mathcal{Q})=\sum_{i \in \mathcal{Q}} R_{i}, \text { for all } \mathcal{Q} \subset \mathcal{M}
$$

The capacity region of an AWGN channel for any given channel vector $\mathbf{h}$ and transmit power constraint vector $\mathbf{P}$ is given by

$$
C(\mathbf{h}, \mathbf{P})=\left\{\mathbf{R}: \mathbf{R}(\mathcal{Q}) \leq \frac{1}{2} \log \left(1+\frac{\sum_{i \in \mathcal{Q}} h_{i} P_{i}}{\sigma^{2}}\right) \forall \mathcal{Q} \subset \mathcal{M}\right\}
$$

We will assume, as in Section II-A, that the number of channel uses $N$ in one slot is large enough that a code of length $N$ with rate close to $C($.) can be used for the needed BER. Given a rate vector $\mathbf{c}$, the set of received powers that can support $\mathbf{c}$ is

$$
P_{r}(\mathbf{h}, \mathbf{c})=\left\{\mathbf{q}: \exists \mathbf{P} \text { s.t } q_{i}=h_{i} P_{i}, \mathbf{c} \in C(\mathbf{h}, \mathbf{P})\right\}
$$

Lemma 2: [15] $C(\mathbf{h}, \mathbf{P})$ is a polymatroid and $P_{r}(\mathbf{h}, \mathbf{c})$ is a contrapolymatroid.

\section{B. Formulation of the Problem as an MDP}

The objective is to minimize the weighted sum of mean delays of $M$ users. Since, the delay process is complicated, as in previous sections, we try to minimize the weighted sum of mean queue lengths. Thus we obtain an optimal policy which minimizes

$$
\limsup _{M \rightarrow \infty} \frac{1}{m} E\left[\sum_{n=0}^{m} \mathbf{w} \cdot \mathbf{S}_{n}\right]
$$

subject to the constraints

$$
\begin{gathered}
\limsup _{M \rightarrow \infty} \frac{1}{m} E\left[\sum_{n=0}^{m} P\left(H_{n}(i), U_{n}(i)\right)\right] \leq \bar{P}_{i} \text { and } \\
\limsup _{M \rightarrow \infty} \frac{1}{m} E\left[\sum_{n=0}^{m} D\left(\frac{A_{i}(n)}{J_{i}}\right)\right] \leq \bar{D}_{i}
\end{gathered}
$$

where $J_{i}$ is the number of source symbols per slot for user $i$ and $\mathbf{w}=$ $\left[w_{1}, \ldots, w_{M}\right]$ is a weight vector. For vectors $\mathbf{a}=\left[a_{1}, \ldots, a_{M}\right]$ and $\mathbf{b}=\left[b_{1}, \ldots, b_{M}\right], \mathbf{a} \cdot \mathbf{b}=\sum_{i=1}^{M} a_{i} b_{i}$.

This constrained $(C P)$ problem can be converted to an unconstrained one through the Lagrange multipliers.

For a state $\mathbf{x}:=(\mathbf{s}, \mathbf{h})$ and the action $\mathbf{r}:=(\mathbf{a}, \mathbf{u})$, let

$$
L(\mathbf{x}, \mathbf{r})=\mathbf{w} . \mathbf{s}+\mathbf{P}_{m}(\boldsymbol{\beta}, \mathbf{u}, \mathbf{h})+\boldsymbol{\theta} \cdot \mathbf{D}(\mathbf{a})
$$

where, $\boldsymbol{\beta}=\left[\beta_{1}, \ldots, \beta_{M}\right]$ and $\boldsymbol{\theta}=\left[\theta_{1}, \ldots, \theta_{M}\right]$ are Lagrange multipliers and $\mathbf{P}(\mathbf{h}, \mathbf{u})=\left[P_{1}\left(\mathbf{h}, u_{1}\right), \ldots, P_{M}\left(\mathbf{h}, u_{M}\right)\right]$, $\mathbf{D}(\mathbf{a})=\left[D_{1}\left(a_{1}\right), \ldots, D_{M}\left(a_{M}\right)\right]$. Also, $\mathbf{P}_{m}$ is the minimum weighted power incurred for the given transmission rates

$$
\mathbf{P}_{m}(\boldsymbol{\beta}, \mathbf{u}, \mathbf{h})=\min _{\mathbf{P}(\mathbf{h}, \mathbf{u})} \sum_{i=1}^{M} \beta_{i} \mathbf{P}\left(\mathbf{h}, u_{i}\right),
$$

subject to $\mathbf{u} \in C(\mathbf{h}, \mathbf{P}(\mathbf{h}, \mathbf{u}))$.
Define the corresponding Lagrangean functional

$$
J^{\pi}=\limsup _{M \rightarrow \infty} \frac{1}{m} E\left[\sum_{n=0}^{m} L\left(\mathbf{X}_{n}, \mathbf{R}_{n}\right]\right.
$$

for a given policy $\pi$. The corresponding unconstrained problem is

$$
(U P) \text { : Minimize } J^{\pi} \text { over the class of all policies } \pi \text {. }
$$

As it was with SISO case, the multiple user model considered also satisfies the nominal conditions given in Lemma 1 and Theorem 2 required for the existence of discount optimal and average optimal stationary policies.

We first obtain $\mathbf{P}_{m}(\boldsymbol{\beta}, \mathbf{u}, \mathbf{h})$. We know that under the successive decoding scheme, the decoding order corresponding to the power allocation policy given by Tse and Hanly [15] is optimal.

The problem (35) can be restated in terms of the received power vector $\mathbf{q}=\left[q_{1}, q_{2}, \ldots, q_{M}\right]=\left[h_{1} P_{1}, \ldots, h_{M} P_{M}\right]$ as

$$
\mathbf{P}_{m}(\boldsymbol{\beta}, \mathbf{u}, \mathbf{h})=\min _{\mathbf{q} \in P_{r}\left(\mathbf{h}, \frac{1}{N}, \mathbf{u}\right)} \sum_{i=1}^{M} \frac{\beta_{i}}{h_{i}} q_{i}
$$

where $P_{r}\left(\mathbf{h}, \frac{1}{N} \mathbf{u}\right)$ is the feasible power region defined by the set of received powers for the given rate vector $\mathbf{u}$ as

$$
\begin{aligned}
P_{r}\left(\mathbf{h}, \frac{1}{N} \mathbf{u}\right) & =\left\{\mathbf{q}: q_{i}=h_{i} P_{i}, \frac{1}{N} \cdot \mathbf{u} \in C(\mathbf{h}, \mathbf{P}(\mathbf{h}, \mathbf{u}))\right\} \\
& =\left\{\mathbf{q}: q_{i}=h_{i} P_{i}, \sum_{k \in Q} q_{k} \geq g(Q) \text { for all } Q \subseteq \mathcal{M}\right\}
\end{aligned}
$$

where $g(Q)=\sigma^{2}\left[\exp \left(v \sum_{k \in Q} u_{k}{ }^{*}\right)-1\right]$ and $v=\frac{2 \ln 2}{N}$. We know that $P_{r}\left(\mathbf{h}, \frac{1}{N} \mathbf{u}\right)$ is a contra-polymatroid with the rank function $g(\cdot)$

We observe that (35) is a linear programming problem. It follows that the optimal solution to the above problem corresponds to a vertex of the region $P_{r}(\mathbf{h}, \mathbf{c})$. Each vertex of the region corresponds to one of $M$ ! possible successive decoding orders, with powers such that the rate $\mathbf{c}$ is achievable. A vertex $\mathbf{q}$ corresponding to successive decoding order $\pi$ is given by

$$
q_{\pi(k)}= \begin{cases}\sigma^{2}\left[\exp \left(v c_{\pi(1)}\right)-1\right], & \text { if } k=1 \\ \sigma^{2}\left[\exp \left(v \sum_{i=1}^{k} c_{\pi(i)}\right)\right. & \\ \left.-\exp \left(v \sum_{i=1}^{k-1} c_{\pi(i)}\right)\right], & \text { if } k=2, \ldots, M .\end{cases}
$$

Here, the decoding order $\pi$ is such that user $\pi(M)$ is decoded first and user $\pi(1)$ decoded last, i.e., $\{\pi(i)\}_{i=1}^{M}$ is decreasing in decoding order.

Since $P_{r}(\mathbf{h}, \mathbf{c})$ is a contra-polymatroid, it follows that the optimal decoding order should be in increasing value of the coefficients $\frac{\beta_{i}}{h_{i}}$, i.e., the user with the smallest $\frac{\beta_{i}}{h_{i}}$ is decoded first and the user with the highest $\frac{\beta_{i}}{h_{i}}$ is decoded last. It is important to note that the optimal ordering does not depend on the target rate vector $\mathbf{c}$, although optimal powers do.

Now, the value iteration algorithm is given by (37) shown at the bottom of the page, where $\pi$ is the optimal permutation order for $\frac{\beta}{\mathbf{h}}=$ $\left(\frac{\beta_{1}}{h_{1}}, \ldots, \frac{\beta_{M}}{h_{M}}\right)$ and $f(x)=\sigma^{2}[\exp (v x)-1]$.

\section{Structural Results}

We obtain some structural results on the optimal cost and policies. We say a vector $\mathbf{x}$ is greater than or equal to a vector $\mathbf{y}$ if the inequality holds for every component, i.e., $\mathbf{x} \geq \mathbf{y} \Rightarrow x_{i} \geq y_{i}$ for all $i$.

Proposition 5: $J_{\alpha}(\mathbf{s}, \mathbf{h})$ is non-decreasing in $\mathbf{s}$ for all $\mathbf{h} \in \mathcal{H}^{M}$.

$$
J_{n+1}(\mathbf{x})=\min _{\mathbf{r}=(\mathbf{a}, \mathbf{u})}\left\{\sum_{k=1}^{M}\left(w_{k} s_{k}+\frac{\beta_{\pi(k)}}{h_{\pi(k)}}\left[f\left(\sum_{j=1}^{k} u_{\pi(j)}\right)-f\left(\sum_{j=1}^{k-1} u_{\pi(j)}\right)\right]+\theta_{k} D\left(a_{k}\right)\right)+\int_{\mathcal{H}^{M}} J_{n}\left(\mathbf{s}-\mathbf{u}+\mathbf{a}, \mathbf{h}^{\prime}\right) d P_{G}\left(\mathbf{h}^{\prime} \mid \mathbf{h}\right)\right\}
$$


Proof: This can be proved by induction on the discounted value iteration algorithm

$$
\begin{aligned}
J_{n+1}(\mathbf{s}, \mathbf{h})=\min _{\mathbf{r}=(\mathbf{a}, \mathbf{u})}\left\{\mathbf{w} \cdot \mathbf{s}+\frac{\boldsymbol{\beta}_{\boldsymbol{\pi}}}{\mathbf{h}_{\boldsymbol{\pi}}} \cdot \mathbf{q}_{\boldsymbol{\pi}}+\boldsymbol{\theta} \cdot D(\mathbf{a})\right. \\
\left.\quad+\alpha \int_{\mathcal{H} M} J_{n}\left(\mathbf{s}-\mathbf{u}+\mathbf{a}, \mathbf{h}^{\prime}\right) d P_{G}\left(\mathbf{h}^{\prime} \mid \mathbf{h}\right)\right\}
\end{aligned}
$$

where, $\alpha \in(0,1), \pi$ is the optimal permutation and

$$
\frac{\beta_{\boldsymbol{\pi}}}{\mathbf{h}_{\boldsymbol{\pi}}} \cdot \mathbf{q}_{\boldsymbol{\pi}}=\frac{\beta_{\pi(k)}}{h_{\pi(k)}}\left[f\left(\sum_{j=1}^{k} u_{\pi(j)}\right)-f\left(\sum_{j=1}^{k-1} u_{\pi(j)}\right)\right] .
$$

For $n=0$ we have $J_{0}(\mathbf{s}, \mathbf{h})=$ constant. Assume $J_{n}(\mathbf{s}, \mathbf{h})$ is nondecreasing in $\mathbf{s}$ for each $\mathbf{h}$ for some $n \geq 0$. We show it for $n+1$. Fix g. Let $\mathbf{s}^{\mathbf{1}}$ and $\mathbf{s}^{\mathbf{2}}$ be such that $\mathbf{s}^{\mathbf{2}} \geq \mathbf{s}^{\mathbf{1}}$. Let $\left(\mathbf{u}^{\mathbf{1}}, \mathbf{a}^{\mathbf{1}}\right)$ and $\left(\mathbf{u}^{\mathbf{2}}, \mathbf{a}^{\mathbf{2}}\right)$ be the corresponding optimal policies. Thus for any $k$

$$
\begin{aligned}
J_{n+1}\left(\mathbf{s}^{k}, h\right)=\mathbf{w} \cdot \mathbf{s}^{k}+ & \frac{\boldsymbol{\beta}_{\pi}}{\mathbf{h}_{\pi}} \cdot \mathbf{q}_{\pi}^{k}+\boldsymbol{\theta} \cdot D\left(\mathbf{a}^{k}\right) \\
& +\alpha \int_{\mathcal{H}^{M}} J_{n}\left(\mathbf{s}^{k}-\mathbf{u}^{k}+\mathbf{a}^{k}, \mathbf{h}^{\prime}\right) d P_{G}\left(\mathbf{h}^{\prime} \mid \mathbf{h}\right)
\end{aligned}
$$

$k=1,2$

We apply policy $\mathbf{r}^{2}=\left(\mathbf{a}^{2}, \mathbf{u}^{2}\right)$ for the state $\mathbf{s}^{1}$. Hence we have the cost for $\mathbf{s}^{1}$ as

$$
\begin{aligned}
J_{n+1}\left(\mathbf{s}^{1}, \mathbf{h}\right) \leq & \mathbf{w} \cdot \mathbf{s}^{1}+\frac{\boldsymbol{\beta}_{\pi}}{\mathbf{h}_{\pi}} \cdot \mathbf{q}_{\pi}^{2}+\boldsymbol{\theta} \cdot D\left(\mathbf{a}^{2}\right) \\
& +\alpha \int_{\mathcal{H} M} J_{n}\left(\mathbf{s}^{1}-\mathbf{u}^{2}+\mathbf{a}^{2}, \mathbf{h}^{\prime}\right) d P_{G}\left(\mathbf{h}^{\prime} \mid \mathbf{h}\right) \\
\leq & \mathbf{w} \cdot \mathbf{s}^{2}+\frac{\boldsymbol{\beta}_{\pi}}{\mathbf{h}_{\pi}} \cdot \mathbf{q}^{2}+\boldsymbol{\theta} \cdot D\left(\mathbf{a}^{2}\right) \\
& +\alpha \int_{\mathcal{H} M} J_{n}\left(\mathbf{s}^{2}-\mathbf{u}^{2}+\mathbf{a}^{2}, \mathbf{h}^{\prime}\right) d P_{G}\left(\mathbf{h}^{\prime} \mid \mathbf{h}\right) \\
= & J_{n+1}\left(\mathbf{s}^{2}, \mathbf{h}\right)
\end{aligned}
$$

where the first inequality follows from the optimality of policies and the second one follows since $\mathbf{s}^{2} \geq \mathbf{s}^{1}$ and the assumption that $J_{n}(., \mathbf{h})$ is nondecreasing. Note that the permutation of decoding priority $\pi$ is same in both the cases since $\mathbf{h}$ is held constant. Hence, $J_{\alpha}(., \mathbf{h})$ is nondecreasing.

Proposition 6: $J_{\alpha}(\mathbf{s}, \mathbf{h})$ is nonincreasing in $\mathbf{h}$ for all $\mathbf{s} \in \mathcal{S}$.

Proof: As above, we prove the proposition by induction on the value iteration algorithm (38).

For $n=0$ we have $J_{0}(\mathbf{s}, \mathbf{h})=$ constant. Assume $J_{n}(\mathbf{s}, \mathbf{h})$ is nonincreasing in $\mathbf{h}$ for each $\mathbf{s}$. Fix $\mathbf{s}$. Let $\mathbf{h}^{1}$ and $\mathbf{h}^{2}$ be the two states such that $\mathbf{h}^{2}>\mathbf{h}^{1}$. Let $\left(\mathbf{u}^{1}, \mathbf{a}^{1}\right)$ and $\left(\mathbf{u}^{2}, \mathbf{a}^{2}\right)$ be the corresponding optimal policies and $\pi$ and $\pi^{\prime}$ be the corresponding optimal decoding orders. Thus

$$
\begin{aligned}
J_{n+1}\left(\mathbf{s}, \mathbf{h}^{2}\right)= & \mathbf{w} \cdot \mathbf{s}+\frac{\boldsymbol{\beta}_{\boldsymbol{\pi}}}{\mathbf{h}^{2}}{ }_{\pi^{\prime}} \cdot \mathbf{q}_{\pi^{\prime}}^{2}+\theta \cdot D\left(\mathbf{a}^{2}\right) \\
& +\alpha \int_{\mathcal{H}^{M}} J_{n}\left(\mathbf{s}-\mathbf{u}^{2}+\mathbf{a}^{2}, \mathbf{h}^{\prime}\right) d P_{G}\left(\mathbf{h}^{\prime} \mid \mathbf{h}^{2}\right) \\
J_{n+1}\left(\mathbf{s}, \mathbf{h}^{1}\right)= & \mathbf{w} \cdot \mathbf{s}+\frac{\boldsymbol{\beta}_{\boldsymbol{\pi}}}{\mathbf{h}^{1}} \cdot \mathbf{q}_{\pi}^{1}+\boldsymbol{\theta} \cdot D\left(\mathbf{a}^{1}\right) \\
& +\alpha \int_{\mathcal{H}^{M}} J_{n}\left(\mathbf{s}-\mathbf{u}^{\mathbf{1}}+\mathbf{a}^{\mathbf{1}}, \mathbf{h}^{\prime}\right) d P_{G}\left(\mathbf{h}^{\prime} \mid \mathbf{h}^{\mathbf{1}}\right) .
\end{aligned}
$$

We apply policy $\mathbf{r}^{1}=\left(\mathbf{a}^{1}, \mathbf{u}^{1}\right)$ with the decoding order $\pi$ for the state $\mathbf{h}^{2}$. Then

$$
\begin{aligned}
J_{n+1}\left(\mathbf{s}, \mathbf{h}^{2}\right) \leq & \mathbf{w} \cdot \mathbf{s}+\frac{\boldsymbol{\beta}_{\boldsymbol{\pi}}}{\mathbf{h}_{\boldsymbol{\pi}}^{2}} \cdot \mathbf{q}_{\boldsymbol{\pi}}^{\mathbf{1}}+\boldsymbol{\theta} \cdot D\left(\mathbf{a}^{\mathbf{1}}\right) \\
& +\alpha \int_{\mathcal{H}^{M}} J_{n}\left(\mathbf{s}-\mathbf{u}^{1}+\mathbf{a}^{1}, \mathbf{h}^{\prime}\right) d P_{G}\left(\mathbf{h}^{\prime} \mid \mathbf{h}^{2}\right) \\
\leq & \mathbf{w} \cdot \mathbf{s}+\frac{\boldsymbol{\beta}_{\boldsymbol{\pi}}}{\mathbf{h}_{\pi}^{\mathbf{1}}} \cdot \mathbf{q}_{\pi}^{\mathbf{1}}+\boldsymbol{\theta} \cdot D\left(\mathbf{a}^{\mathbf{1}}\right) \\
& +\alpha \int_{\mathcal{H}^{M}} J_{n}\left(\mathbf{s}-\mathbf{u}^{1}+\mathbf{a}^{1}, \mathbf{h}^{\prime}\right) d P_{G}\left(\mathbf{h}^{\prime} \mid \mathbf{h}^{2}\right)
\end{aligned}
$$

where the first inequality follows from the optimality of policies and the second one follows since $\mathbf{h}^{1}>\mathbf{h}^{2}$. Now we use the fact that the fading process of each user $\left\{H_{n}(i), i=1, \ldots, M\right\}$ is stochastically monotone and $J_{n}($.$) is nonincreasing in \mathbf{h}$. Therefore

$$
\begin{aligned}
& \int_{\mathbf{h}^{\prime} \in \mathcal{H} \mathbf{M}} J_{n}\left(\mathbf{s}, \mathbf{h}^{\prime}\right) d P_{G}\left(\mathbf{h}^{\prime} \mid \mathbf{h}^{2}\right) \\
& =\int_{h_{1}^{\prime} \in \mathcal{H}} \cdots \int_{h^{\prime}{ }_{M} \in \mathcal{H}} J_{n}\left(\mathbf{s}, \mathbf{h}^{\prime}\right) d P_{G}\left(h_{1}^{\prime} \mid h_{1}^{2}\right) \ldots d P_{G}\left(h_{M}^{\prime} \mid h_{M}^{2}\right) \\
& \leq \int_{h_{1}^{\prime} \in \mathcal{H}} \cdots \int_{h_{M}^{\prime} \in \mathcal{H}} J_{n}\left(\mathbf{s}, \mathbf{h}^{\prime}\right) d P_{G}\left(h_{1}^{\prime} \mid h_{1}^{1}\right) \ldots d P_{G}\left(h_{M}^{\prime} \mid h_{M}^{1}\right) \\
& =\int_{\mathbf{h}^{\prime} \in \mathcal{H} \mathbf{M}} J_{n}\left(\mathbf{s}, \mathbf{h}^{\prime}\right) d P_{G}\left(\mathbf{h}^{\prime} \mid \mathbf{h}^{1}\right) .
\end{aligned}
$$

Thus we have

$$
\begin{aligned}
\int_{\mathbf{h}^{\prime} \in \mathcal{H}^{\mathrm{M}}} J_{n}\left(\mathbf{s}-\mathbf{u}^{1}+\right. & \left.\mathbf{a}^{1}, \mathbf{h}^{\prime}\right) d P_{G}\left(\mathbf{h}^{\prime} \mid \mathbf{h}^{2}\right) \\
& \leq \int_{\mathbf{h}^{\prime} \in \mathcal{H} \mathbf{M}} J_{n}\left(\mathbf{s}-\mathbf{u}^{1}+\mathbf{a}^{1}, \mathbf{h}^{\prime}\right) d P_{G}\left(\mathbf{h}^{\prime} \mid \mathbf{h}^{1}\right)
\end{aligned}
$$

and hence, from (39), we get

$$
J_{n+1}\left(\mathbf{s}, \mathbf{h}^{2}\right) \leq J_{n+1}\left(\mathbf{s}, \mathbf{h}^{1}\right) .
$$

\section{A Suboptimal Policy}

The optimal policy provided above will be computed via the value iteration algorithm. The computational complexity of this algorithm for multiuser case is very high. Also, since it is explicitly not available, except the above mentioned structural results, we do not have any insight. Therefore, we explore a suboptimal policy which is much easier to compute and can be easily explained.

From the value iteration algorithm on the average cost policy

$$
\begin{aligned}
J_{2}(\mathbf{s}, \mathbf{h})= & \min _{(\mathbf{a}, \mathbf{u})}\left\{\mathbf{w} \cdot \mathbf{s}+\mathbf{P}_{m}(\boldsymbol{\beta}, \mathbf{u}, \mathbf{h})+\boldsymbol{\theta} \cdot(D(\mathbf{a})\right. \\
& +D(\bar{A}))+\mathbf{w} \cdot(\mathbf{s}-\mathbf{u}+\mathbf{a})\} \\
= & \min _{u}\left\{\mathbf{P}_{m}(\boldsymbol{\beta}, \mathbf{u}, \mathbf{h})-\mathbf{w} \cdot \mathbf{u}\right\} \\
& +\min _{a}\{\boldsymbol{\theta} \cdot(D(\mathbf{a})+\mathbf{w} \cdot \mathbf{a}\}+K
\end{aligned}
$$

where $K=2 \mathbf{w} \cdot \mathbf{s}+\theta \cdot D(\overline{\mathbf{A}})$. This is a much simpler problem because it has been separated into two optimization problems. Also, it is intuitively easy to explain.

Consider the problem of minimizing $V_{1}=\boldsymbol{\theta} \cdot D(\mathbf{a})+\mathbf{w} \cdot \mathbf{a}=$ $\sum_{i=1}^{M}\left(\theta_{i} D\left(a_{i}\right)+w_{i} a_{i}\right)$. The optimization can be done separately for each user. If $D$ is differentiable, then $a_{i}$ solving $D^{\prime}\left(a_{i}\right)=-\frac{w_{i}}{\theta_{i}}$ 


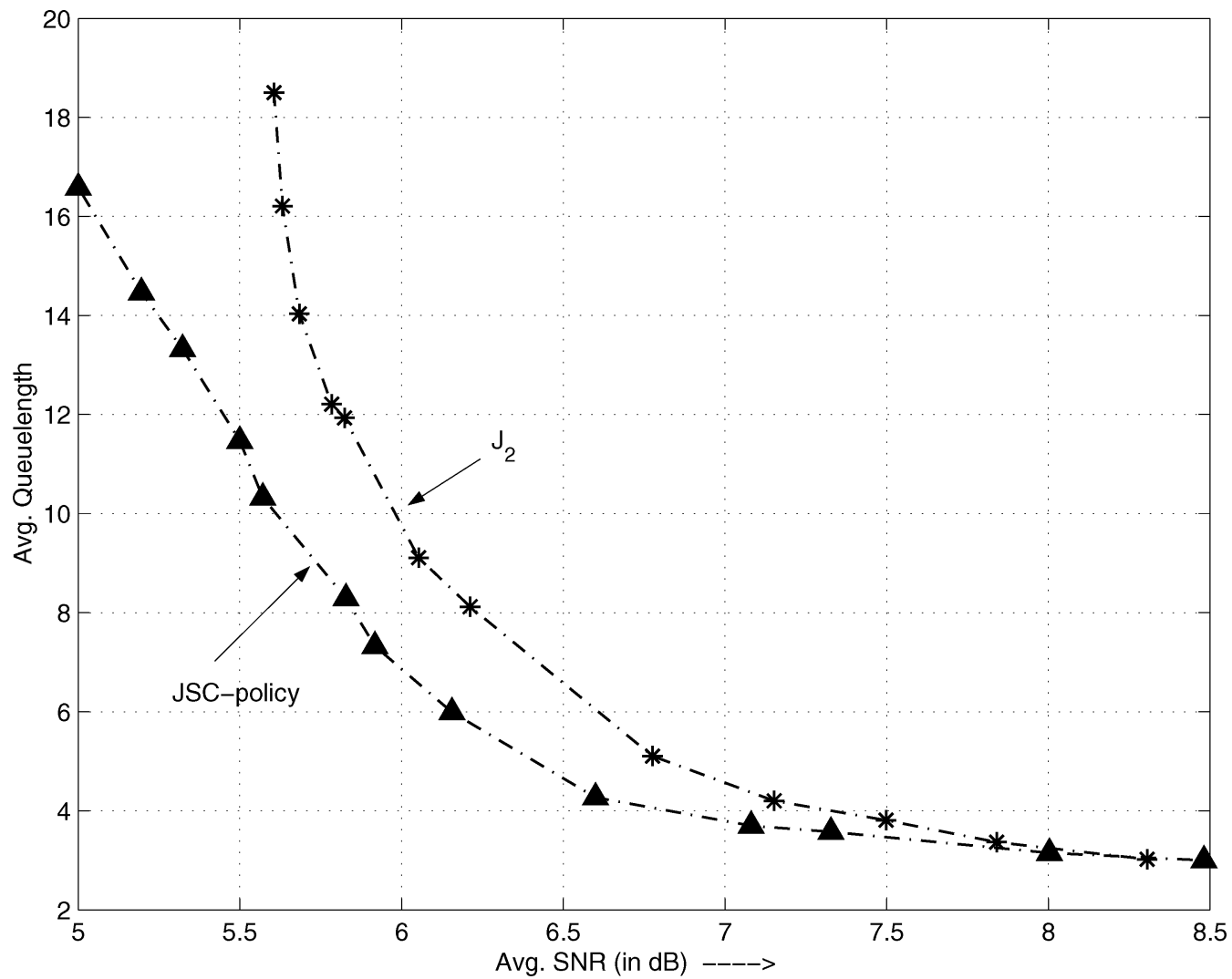

Fig. 4. Average buffer length versus average power for JSC policy.

provides the optimal solution. For example, for Gaussian sources, $D\left(a_{i}\right)=\sigma_{s}^{2} 2^{\left(-2 a_{i}\right)}$ and the solution is

$$
a_{i}=\left\lfloor\frac{1}{k} \ln \left(\frac{k \theta_{i} \sigma_{s}^{2}}{w_{i}}\right)\right\rfloor^{+}, k=2 \ln (2) .
$$

The solution says that fix the source coding rate for user $i$ according to the value of $\theta_{i}$ and $w_{i}$. A user with higher value of $\frac{\theta_{i}}{w_{i}}$ will have higher compression rate indicating that the distortion requirements are more stringent than delay requirements.

Now, consider the problem of minimizing

$$
V_{2}=\left\{\mathbf{P}_{m}(\boldsymbol{\beta}, \mathbf{u}, \mathbf{h})-\mathbf{w} . \mathbf{u}\right\} \text { over } \mathbf{0} \leq \mathbf{u} \leq \mathbf{s} .
$$

To solve this constrained problem we introduce Lagrange multipliers $\lambda_{i}, \nu_{i}, i=1,2 \ldots, M$ and the corresponding Lagrangean is

$$
L(\mathbf{u}, \boldsymbol{\lambda}, \boldsymbol{\nu})=\mathbf{P}_{m}(\boldsymbol{\beta}, \mathbf{u}, \mathbf{h})-\mathbf{w} . \mathbf{u}+\boldsymbol{\lambda} . \mathbf{u}-\boldsymbol{\nu} . \mathbf{u} .
$$

For simplicity, we consider the case $M=2$. Let $\mu_{i}=\frac{\beta_{i}}{h_{i}}, i=$ 1,2 . Without loss of generality we can assume that $\mu_{1}>\mu_{2}$. The Lagrangean is

$$
\begin{aligned}
L(\mathbf{u}, \boldsymbol{\lambda}, \boldsymbol{\nu})=\mu_{1}\left(e^{v u_{1}}-1\right) & +\mu_{2}\left(e^{v\left(u_{1}+u_{2}\right)}-e^{v u_{1}}\right) \\
& +\left(\lambda_{1}-\nu_{1}-w_{1}\right) u_{1}+\left(\lambda_{1}-\nu_{1}-w_{1}\right) u_{2}
\end{aligned}
$$

where $v=\frac{2 \ln (2)}{N}$.

Taking the derivatives of $L($.$) with respect to u_{1}$ and $u_{2}$ and setting them to zero, we get

$$
\begin{array}{r}
v\left(\mu_{1}-\mu_{2}\right) e^{v u_{1}}+v \mu_{2} e^{v\left(u_{1}+u_{2}\right)}+\left(\lambda_{1}-\nu_{1}-w_{1}\right)=0 \\
v \mu_{2} e^{v\left(u_{1}+u_{2}\right)}+\left(\lambda_{2}-\nu_{2}-w_{2}\right)=0 .
\end{array}
$$

We find the values of $u_{1}, u_{2}$ that satisfy these equations along with Kuhn-Tucker (KKT) conditions.
We consider the following cases

- Case 1) $w_{1}>w_{2}$.

The solution pairs satisfying KKT conditions are

$$
\begin{aligned}
& \left\{u_{1}=0, u_{2}=0\right\} \\
& \left\{u_{1}=\min \left(\left\lfloor\frac{1}{v} \ln \left(\frac{w_{1}-w_{2}}{v\left(\mu_{1}-\mu_{2}\right)}\right)\right\rfloor^{+}, s_{1}\right)\right. \\
& \left.u_{2}=\min \left(\left\lfloor\frac{1}{v} \ln \left(\frac{w_{2}\left(\mu_{1}-\mu_{2}\right)}{\mu_{2}\left(w_{1}-w_{2}\right)}\right)\right\rfloor^{+}, s_{2}\right)\right\} \\
& \left\{u_{1}=\min \left(\left\lfloor\frac{1}{v} \ln \left(\frac{w_{1}}{v\left(\mu_{1}-\mu_{2}+\mu_{2} e^{\left.v s_{2}\right)}\right.}\right)\right\rfloor^{+}, s_{1}\right), u_{2}=s_{2}\right\} \\
& \left\{u_{1}=0, u_{2}=\min \left(\left\lfloor\left[\frac{1}{v} \ln \left(\frac{w_{2}}{v \mu_{2}}\right)\right\rfloor^{+}, s_{2}\right)\right\}\right. \\
& \left\{u_{1}=0, u_{2}=s_{2}\right\} \\
& \left\{u_{1}=s_{1}, u_{2}=s_{2}\right\} .
\end{aligned}
$$

For a given state $(\mathbf{s}, \mathbf{h})$, among the above solution pairs we should choose the one for which the value of $V_{2}$ is minimum.

- Case 2) $w_{1} \leq w_{2}$.

The solution pairs satisfying KKT conditions are

$$
\begin{aligned}
& \left\{u_{1}=0, u_{2}=0\right\} \\
& \left\{u_{1}=\min \left(\left\lfloor\frac{1}{v} \ln \left(\frac{w_{1}}{v\left(\mu_{1}-\mu_{2}+\mu_{2} e^{v s_{2}}\right)}\right)\right\rfloor^{+}, s_{1}\right), u_{2}=s_{2}\right\} \\
& \left\{u_{1}=0, u_{2}=\min \left(\left\lfloor\frac{1}{v} \ln \left(\frac{w_{2}}{v \mu_{2}}\right)\right\rfloor^{+}, s_{2}\right)\right\} \\
& \left\{u_{1}=s_{1}, u_{2}=s_{2}\right\} \\
& \left\{u_{1}=0, u_{2}=s_{2}\right\} .
\end{aligned}
$$

For a given state $(\mathbf{s}, \mathbf{h})$, among the above solution pairs we should choose the one for which the value of $V_{2}$ is minimum. 


$$
\begin{aligned}
J_{n+1}(s-1, g)+J_{n+1}(s+1, g) \\
=2 s+\beta P\left(g, u_{1}\right)+\beta P\left(g, u_{2}\right)+\theta D\left(a_{1}\right)+\theta D\left(a_{2}\right) \\
+\alpha \int_{g^{\prime} \in \mathcal{G}}\left(J_{n}\left(s+1-u_{1}+a_{1}, g^{\prime}\right)+J_{n}\left(s-1-u_{2}+a_{2}, g^{\prime}\right)\right) d P_{G}\left(g^{\prime} \mid g\right) \\
\geq \beta\left(P\left(g,\left\lceil\frac{u_{1}+u_{2}}{2}\right\rceil\right)+P\left(g,\left\lfloor\frac{u_{1}+u_{2}}{2}\right\rfloor\right)\right)+\theta\left(D\left(\left\lceil\frac{a_{1}+a_{2}}{2}\right\rceil\right)+D\left(\left\lfloor\frac{a_{1}+a_{2}}{2}\right\rfloor\right)\right) \\
+\alpha \int_{g^{\prime} \in \mathcal{G}}\left(J_{n}\left(\left\lceil\frac{\left(s+1-u_{1}+a_{1}\right)+\left(s-1-u_{2}+a_{2}\right)}{2}\right\rceil, g^{\prime}\right)+J_{n}\left(\left\lfloor\frac{\left(s+1-u_{1}+a_{1}\right)+\left(s-1-u_{2}+a_{2}\right)}{2}\right\rfloor, g^{\prime}\right)\right) d P_{G}\left(g^{\prime} \mid g\right) \\
=\beta\left(P\left(g,\left\lceil\frac{u_{1}+u_{2}}{2}\right\rceil\right)+P\left(g,\left\lfloor\frac{u_{1}+u_{2}}{2}\right\rfloor\right)\right)+\theta\left(D\left(\left\lceil\frac{a_{1}+a_{2}}{2}\right\rceil\right)+D\left(\left\lfloor\frac{a_{1}+a_{2}}{2}\right\rfloor\right)\right) \\
\quad+\alpha \int_{g^{\prime} \in \mathcal{G}}\left(J_{n}\left(s-\left\lfloor\frac{\left(u_{1}+u_{2}\right)}{2}\right\rfloor+\left\lceil\frac{\left(a_{1}+a_{2}\right)}{2}\right\rceil, g^{\prime}\right)+J_{n}\left(s-\left\lceil\frac{\left(u_{1}+u_{2}\right)}{2}\right\rceil+\left\lfloor\frac{\left(a_{1}+a_{2}\right)}{2}\right\rfloor, g^{\prime}\right)\right) d P_{G}\left(g^{\prime} \mid g\right) \\
\geq 2 J_{n+1}(s, g) .
\end{aligned}
$$

For comparison, we consider a two user MAC system with symmetry in all requirements. The fading of each user is a four-state i.i.d. process. The number of channel uses per slot, $N$ is 5 for each user. The sources are i.i.d. and the channel noise is AWGN with $W \sim \mathcal{N}(0,1)$ for each user. The comparison of $J_{2}$ with the optimal JSC-policy is shown in Fig. 4 for a fixed average distortion $(=1.5 \%)$. JSC-policy performs better than $J 2$. However, above $7.5 \mathrm{~dB}$, the two policies are comparable.

\section{CONCLUSIONS AND Future DiRECTIONS}

In this correspondence, we considered the problem of joint source and channel coding for a wireless link with the goal of minimizing the mean queue length. We proved the existence of optimal stationary policies. We considered a single user with SISO and MIMO channel and multiuser systems. We also characterized the structure of the optimal policies and the value function. Our scheme achieved substantial gain in minimizing mean queue length as well as mean delay when compared to the other suboptimal schemes. Thus, our work shows that it is useful to jointly optimize source and channel coding rates exploiting channel and buffer states. We have also shown that using multiple antennas at transmitter and receiver can significantly improve the performance. For the multiuser case we have identified a much simpler policy which provides reasonable performance at not very low SNR.

The work can be extended to the case where only partial channel state information is available at the transmitter. It will be useful to know the effect of partial CSIT. Furthermore, although we are able to give some structure of the optimal policy and a explicitly characterized suboptimal policy in case of multiuser, the behavior of optimal policy needs to be studied further. It will also be useful to obtain better suboptimal policies.

\section{APPENDIX}

\section{PROOF OF THE PROPOSITION 3}

$J_{0}(s, g)$ is constant. Assume $J_{n}(s+1, g)-J_{n}(s, g)$ is nondecreasing in $s$ for each $g$. Fix $g$.

Let $r_{1}=\left(a_{1}, u_{1}\right)$ and $r_{2}=\left(a_{2}, u_{2}\right)$ be optimal policies for states $s+1$ and $s-1$, respectively. Then by value iteration, we have (43) shown at the top of the page.

Inequalities (44) and (45) (also at the top of the page) follow from convexity assumptions on $P(g,),. J_{n}(., g)$ (and the fact that $D($.$) is$ convex [5]) and also the optimality of policies. Therefore, we have

$$
J_{n+1}(s+1, g)-J_{n+1}(s, g) \geq J_{n+1}(s, g)-J_{n+1}(s-1, g) .
$$

Thus $J_{n}(s+1, g)-J_{n}(s, g)$ is an nondecreasing function of $s$.

\section{REFERENCES}

[1] R. A. Berry and R. G. Gallager, "Communication over fading channels with delay constraints," IEEE Trans. Inf. Theory, vol. 48, no. 5, pp. 1135-1149, 2002.

[2] V. Borkar, S. K. Mitter, and S. Tatikonda, "Optimal sequential vector quantization of Markov sources," SIAM J. Contr. Optim., vol. 40, pp. 135-148, 2001.

[3] Y. Eisenberg, C. E. Luna, T. N. Pappas, R. Berry, and A. K. Katsaggelos, "Joint source coding and transmission power management for energy efficient wireless video communications," IEEE Trans. Circuits Syst. Video Technol., vol. 12, pp. 411-424, 2002.

[4] M. Goyal, A. Kumar, and V. Sharma, "Power constrained and delay optimal policies for scheduling transmission over a fading channel," in Proc. IEEE INFOCOM 2003, San Francisco, CA.

[5] R. M. Gray, Entropy and Information Theory. New York: SpringerVerlag, 1990.

[6] O. Hernandez-Lerma and J. B. Lassere, Discrete-Time Markov Control Processes. New York: Springer-Verlag, 1996.

[7] B. Hochwald and K. Zeger, "Trade off between source and channel coding," IEEE Trans. Inf. Theory, vol. 43, pp. 1412-1424, 1997.

[8] H. Jafarkhani, P. Ligdas, and N. Farvardin, "Adaptive rate allocation in a joint source/channel coding framework for wireless channels," in Proc. IEEE VTC'96, Apr. 1996, pp. 492-496.

[9] A. Kashyap, T. Basar, and R. Srikant, "Minimum distortion transmission of Gaussian sources over fading channels," in Proc. 42nd IEEE Conf. Decision and Control, 2003, pp. 80-85.

[10] R. L. Martinez, "A saddle-point theorem for constrained Markov control processes," Morfismos, vol. 3, no. 2, 1999.

[11] D. J. Ma, A. M. Makowski, and A. Schwartz, "Estimation and optimal control for constrained Markov chains," in Proc. 25th IEEE Conf. Dec. Contr., 1986, pp. 994-999.

[12] A. Paulraj, R. Nabar, and D. Gore, Introduction to Space-Time Wireless Communications. New York: Cambridge University Press, 2003.

[13] M. Schal, "Average optimality in dynamic programming with general state space," Math. Oper. Res. , vol. 18, no. 1, pp. 163-172, 1993.

[14] S. Vembu, S. Verdu, and Y. Steinberg, "The source-channel separation theorem revisited," IEEE Trans. Inf. Theory, vol. 41, pp. 44-54, 1995.

[15] D. N. C. Tse and S. V. Hanly, "Multiaccess fading channels-Part I : Polymatroid structure, optimal resource allocation and throughput capacities," IEEE Trans. Inf. Theory, vol. 44, pp. 2796-2830, 1998. 